\title{
LOCAL HARDY AND BMO SPACES ON NON-HOMOGENEOUS SPACES
}

\author{
DACHUN YANG
}

(Received 1 December 2001; revised 7 April 2004)

\author{
Communicated by A. H. Dooley
}

\begin{abstract}
Let $\mu$ be a Radon measure on $\mathbb{R}^{d}$ which may be non doubling. The only condition that $\mu$ must satisfy is the size condition $\mu(B(x, r)) \leq C r^{n}$ for some fixed $n \in(0, d]$. Recently, Tolsa introduced the spaces $R B M O(\mu)$ and $H_{a, b}^{1, \infty}(\mu)$, which, in some ways, play the role of the classical spaces $B M O$ and $H^{1}$ in case $\mu$ is a doubling measure. In this paper, the author considers the local versions of the spaces $R B M O(\mu)$ and $H_{a b b}^{1, \infty}(\mu)$ in the sense of Goldberg and establishes the connections between the spaces $R B M O(\mu)$ and $H_{a t b}^{1, \infty}(\mu)$ with their local versions. An interpolation result of linear operators is also given.
\end{abstract}

2000 Mathematics subject classification: primary 42B35; secondary 42B30, 42B25, 43A99.

Keywords and phrases: local Hardy space, local $B M O$ space, atomic block, block, non-doubling measure, interpolation, duality.

\section{Introduction}

In the whole paper we assume that $d \in \mathbb{N}$ and $0<n \leq d$. We also assume $\mu$ is a (positive) Radon measure on $\mathbb{R}^{d}$ satisfying the growth condition

$$
\mu(B(x, r)) \leq C_{0} r^{n}
$$

for all $x \in \operatorname{supp} \mu$ and $r>0$. We do not assume that $\mu$ is doubling.

This paper was written during the author's visit to the Friedrich-Schiller University at Jena as a Humboldt Research Fellow. He gratefully acknowledges the support of the Alexander von Humboldt Foundation in Germany and the partial support of both the NSF (No. 10271015) and the RFDP (No. 20020027004) of China. Special thanks are due to Professor Hans Triebel, Professor Xavier Tolsa and Professor Yongsheng Han for generously sharing many ideas with him. The author also wants to thank the referee for his/her helpful comments, which made this article more readable.

(C) 2005 Australian Mathematical Society 1446-7887/05 $\$$ A2.00+0.00 
The doubling condition on $\mu$ is an essential assumption in most results of classical function spaces, Calderón-Zygmund theory and so on. However, recently it has been shown that many results in the classical Calderon-Zygmund theory and the classical Hardy and $B M O$ spaces also hold without the doubling assumption; see [8-16] and their references. In particular, Tolsa in [13] introduced the spaces $R B M O(\mu)$ and $H_{a t b}^{1, \infty}(\mu)$ and proved that these spaces have properties similar to ones of the classical spaces $B M O$ and $H^{1}$ defined for doubling measures and are useful in the study of the $L^{p}(\mu)$ boundedness of Calderón-Zygmund operators without assuming doubling conditions; see also $[14,15]$.

The purpose of this paper is to consider the local versions of the spaces $R B M O(\mu)$ and $H_{a r b}^{1, \infty}(\mu)$ in the sense of Goldberg [4] by using ideas coming from [13]. The organization of this paper is as follows. In Section 2, we introduce the local version, $r b m o(\mu)$, of the space $R B M O(\mu)$ in [13] and then give some basic properties including several equivalent definitions of this local space. By using these properties, we establish the John-Nirenberg's inequality for the functions in the space $r b m o(\mu)$. In Section 3 we introduce the local version, $h_{a t b}^{1, \infty}(\mu)$, of the space $H_{a t b}^{1, \infty}(\mu)$ in [13] and we then prove the dual space of the space $h_{a t b}^{1, \infty}(\mu)$ is just the space $r b m o(\mu)$. An interpolation result of linear operators is given in Section 4, which states that if a linear operator $T$ is bounded from $h_{a t b}^{1, \infty}(\mu)$ into $L^{1}(\mu)$ and from $L^{\infty}(\mu)$ into $r b m o(\mu)$, then $T$ is also bounded on $L^{p}(\mu)$ for $p \in(1, \infty)$. In Section 5, we establish the connections between the spaces $R B M O(\mu)$ and $H_{a t b}^{1, \infty}(\mu)$ and their local spaces, $r b m o(\mu)$ and $h_{a t b}^{1, \infty}(\mu)$, respectively. Finally, in Section 6, we give some remarks on our results in case $\mu$ is doubling. Even in this case, our result is also new.

Let us now introduce some basic notation. By a cube $Q \subset \mathbb{R}^{d}$, we mean a closed cube centered at some point in $\operatorname{supp} \mu$ with sides parallel to the axes. Its side length is denoted by $l(Q)$ and its center by $z_{Q}$. Given $\rho>0$, we denote by $\rho Q$ the cube concentric with $Q$ with side length $\rho l(Q)$.

Definition 1.1. Given $\alpha>1$ and $\beta>\alpha^{n}$, we say that the cube $Q \subset \mathbb{R}^{d}$ is $(\alpha, \beta)$-doubling if $\mu(\alpha Q) \leq \beta \mu(Q)$.

As shown in [13], due to the fact that $\mu$ satisfies the growth condition (1.1), there are a lot of 'big' doubling cubes. To be precise, given any point $x \in \operatorname{supp} \mu$ and $c>0$, there exists some $(\alpha, \beta)$-doubling cube $Q$ centered at $x$ with $l(Q) \geq c$, which follows from (1.1) and the fact that $\beta>\alpha^{n}$.

On the other hand, if $\beta>\alpha^{n}$, then for $\mu$-a.e. $x \in \mathbb{R}^{d}$, there exists a sequence of $(\alpha, \beta)$-doubling cubes $\left\{Q_{k}\right\}_{k}$ centered at $x$ with $l\left(Q_{k}\right) \rightarrow 0$ as $k \rightarrow \infty$; see also [13]. So there are a lot of 'small' doubling cubes too.

In the following, for any $\rho>1$, we denote by $\beta_{\rho}$ one of these big constants $\beta$. For definiteness, one can assume that $\beta_{\rho}$ is twice the infimum of these $\beta$ 's.

Recall that given two cubes $Q \subset R, Q_{R}$ stands for the smallest cube concentric 
with $Q$ containing $R$. Without assuming $Q \subset R$, we denote by $Q_{R}$ the smallest cube concentric with $Q$ containing $Q$ and $R$.

Definition 1.2. Consider two cubes $Q, R \subset \mathbb{R}^{d}$. We denote

$$
\delta(Q, R)=\max \left(\int_{Q_{R} \backslash Q} \frac{1}{\left|x-z_{Q}\right|^{n}} d \mu(x), \int_{R_{Q} \backslash R} \frac{1}{\left|x-z_{R}\right|^{n}} d \mu(x)\right) .
$$

Notice that $l\left(Q_{R}\right) \sim l\left(R_{Q}\right) \sim l(Q)+l(R)+\operatorname{dist}(Q, R)$, and if $Q \subset R$, then $R_{Q}=R$ and $l(R) \leq l\left(Q_{R}\right) \leq 2 l(R)$.

$\delta(Q, R)$ was first introduced by Tolsa in [13]; see also $[14,15]$.

Throughout the paper, the letter $C$ is used for non-negative constants that may change from one occurrence to another. Constants with subscripts, such as $C_{0}$, do not change in different occurrences. For any cube $Q \subset R$, we write $K_{Q, R}=1+\delta(Q, R)$.

The following lemma was proved by Tolsa in [13], which plays a fundamental role in the whole theory. From this lemma, it is easy to see that $K_{Q, R}$ reflects some geometric aspects of cubes.

LEMMA 1.3. Let $C \geq 0$ be a constant.

(i) If $Q \subset R \subset S$ are cubes in $\mathbb{R}^{d}$, then $K_{Q, R} \leq K_{Q, S}, K_{R, S} \leq C K_{Q, S}$ and $K_{Q, S} \leq C\left(K_{Q, R}+K_{R, S}\right)$.

(ii) If $Q \subset R$ have comparable sizes, $K_{Q, R} \leq C$.

(iii) If $N$ is some positive integer and the cubes $\rho Q, \rho^{2} Q, \ldots, \rho^{N-1} Q$ are non $(\rho, \beta)$-doubling with $\beta>2^{n}$, then $K_{Q, \rho^{N} Q} \leq C$ with $C$ depending on $\beta, \rho$ and $C_{0}$.

(iv) If $N$ is a positive integer and for some $\beta<\rho^{n}$,

$$
\mu\left(\rho^{N} Q\right) \leq \beta \mu\left(\rho^{N-1} Q\right) \leq \beta^{2} \mu\left(\rho^{N-2} Q\right) \leq \cdots \leq \beta^{N} \mu(Q),
$$

then $K_{Q, \rho^{N} Q} \leq C$ with $C$ depending on $\beta, \rho$ and $C_{0}$.

\section{The space $r b m o(\mu)$}

Given $\rho>1$ and a cube $Q \subset \mathbb{R}^{d}$, we let $N$ be the smallest nonnegative integer such that $2^{N} Q$ is $\left(\rho, \beta_{\rho}\right)$ doubling and we denote this cube by $\widetilde{Q}$. In the following, we also set

$$
m_{Q} f=\frac{1}{\mu(Q)} \int_{Q} f(x) d \mu(x)
$$

DEFINITION 2.1. Let $\rho>1$ be some fixed constant. 
(i) Let $1<\eta<\infty$. We say that $f \in L_{\text {loc }}^{1}(\mu)$ is in $R B M O(\mu)$ if there exists some constant $C_{1} \geq 0$ such that for any cube $Q$,

$$
\frac{1}{\mu(\eta Q)} \int_{Q}\left|f-m_{\tilde{Q}} f\right| d \mu \leq C_{1},
$$

and for any two $\left(\rho, \beta_{\rho}\right)$-doubling cubes $Q \subset R$,

$$
\left|m_{Q} f-m_{R} f\right| \leq C_{1} K_{Q, R} .
$$

Moreover, we define the $R B M O(\mu)$ norm of $f$ by the minimal constant $C_{1}$ and we denote this by $\|\cdot\|_{*}$.

(ii) Let $1<\eta \leq \rho<\infty$. We say that $f \in L_{\text {loc }}^{1}(\mu)$ is in $r b m o(\mu)$ if there exists some constant $C_{2} \geq 0$ such that (2.1) holds for any cube $Q$ with $l(Q) \leq 1$ and $C_{2}$ instead of $C_{1},(2.2)$ holds for any two $\left(\rho, \beta_{\rho}\right)$-doubling cubes $Q \subset R$ with $l(Q) \leq 1$ and $C_{2}$ instead of $C_{1}$, and

$$
\frac{1}{\mu(\eta Q)} \int_{Q}|f(x)| d \mu(x) \leq C_{2}
$$

for any cube $Q$ with $l(Q)>1$. Moreover, we define the $r b m o(\mu)$ norm of $f$ by the minimal constant $C_{2}$ and we denote this by $\|\cdot\|_{*}$.

The space $R B M O(\mu)$ was introduced by Tolsa in [13] and it was proved that the definition of $R B M O(\mu)$ in [13] is independent of the choices of $\eta$ and $\rho$. It is easy to see that $r b m o(\mu) \subset R B M O(\mu)$, and there are some measures $\mu$ (for example, the $d$-dimensional Lebesgue measure) such that the above inclusion is strict.

The following proposition is similar to [13, Proposition 2.5] whose proof is also similar. In fact, the properties (i) and (ii) are easy to check. The third property can easily follows from the following Proposition 2.4 and the fourth property follows from the third one.

PROPOSITION 2.2. (i) $r b m o(\mu)$ is a Banach space of functions (modulo additive constants).

(ii) $L^{\infty}(\mu) \subset \operatorname{rbmo}(\mu)$ with $\|f\|_{*} \leq 2\|f\|_{L^{\infty}(\mu)}$.

(iii) If $f \in \operatorname{rbmo}(\mu)$, then $|f| \in \operatorname{rbmo}(\mu)$ and $\|\mid f\|_{*} \leq C\|f\|_{*}$.

(iv) If $f, g \in \operatorname{rbmo}(\mu)$, then $\min (f, g), \max (f, g) \in \operatorname{rbmo}(\mu)$ and

$$
\|\min (f, g)\|_{*},\|\max (f, g)\|_{*} \leq C\left(\|f\|_{*}+\|g\|_{*}\right) .
$$

Let us now see some other equivalent norms for the space $r b m o(\mu)$. Let $\eta \in(1, \infty)$. Suppose that for a given function $f \in L_{\text {loc }}^{1}(\mu)$, there exist some constant $C_{3} \geq 0$ and 
a collection of numbers $\left\{f_{Q}\right\}_{Q}$ (that is, for each cube $Q$ there exists $f_{Q} \in \mathbb{R}$ ) such that

$$
\begin{gathered}
\sup _{Q: l(Q) \leq 1} \frac{1}{\mu(\eta Q)} \int_{Q}\left|f(x)-f_{Q}\right| d \mu(x) \leq C_{3}, \\
\left|f_{Q}-f_{R}\right| \leq C_{3} K_{Q, R}
\end{gathered}
$$

for any two cubes $Q \subset R$ with $l(Q) \leq 1$, and for any cube $R$ with $l(R)>1$,

$$
\left|f_{R}\right| \leq C_{3} \text {. }
$$

We then write $\|f\|_{* *}=\inf \left\{C_{3}\right\}$, where the infimum is taken over all the constants $C_{3}$ and all the numbers $\left\{f_{Q}\right\}_{Q}$ satisfying (2.4)-(2.6).

Temporarily, we write $\|\cdot\|_{* *, \eta}$ instead of $\|\cdot\|_{* *}$. The following proposition indicates that $\|\cdot\|_{* * . \eta}$ is independent of $\eta$, the proof of it is a slight variation of [13, Lemma 2.6]. We omit the details.

PROPOSITION 2.3. The norms $\|\cdot\|_{* *, \eta}$ are independent of $\eta \in(1, \infty)$.

ReMARK 2.1. Let $\eta \in(1, \infty)$. From the proof of Proposition 2.3 (see the proof of [13, Lemma 2.6]), we can see that if $C_{f} \geq 0$ is some constant and $\left\{f_{Q}\right\}_{Q}$ is some fixed collection of numbers satisfying that for $Q$ with $l(Q) \leq 1$,

$$
\sup _{Q: l(Q) \leq 1} \frac{1}{\mu(\eta Q)} \int_{Q}\left|f(x)-f_{Q}\right| d \mu(x) \leq C_{f}, \quad\left|f_{Q}-f_{R}\right| \leq C_{f} K_{Q, R}
$$

for any two cubes $Q \subset R$ with $l(Q) \leq 1$, and for any cube $R$ with $l(R)>1$, $\left|f_{R}\right| \leq C_{f}$, then for the same numbers $\left\{f_{Q}\right\}_{Q}$ and any $\eta_{1} \in(1, \infty)$, we have

$$
\sup _{Q: I(Q) \leq 1} \frac{1}{\mu\left(\eta_{1} Q\right)} \int_{Q}\left|f(x)-f_{Q}\right| d \mu(x) \leq C C_{f}
$$

with $C \geq 0$ depending on $\eta$ and $\eta_{1}$.

Next proposition proves that for any fixed $\eta \in(1, \infty)$ and $\eta \leq \rho<\infty$, the norms $\|\cdot\|_{* *}$ and $\|\cdot\|_{*}$ are equivalent, which indicates that the norms $\|\cdot\|_{*}$ are also independent of $1<\eta \leq \rho<\infty$.

Proposition 2.4. For a fixed $\eta \in(1, \infty)$ and a fixed $\rho \in[\eta, \infty)$, the norms $\|\cdot\|_{* *}$ and $\|\cdot\|_{*}$ are equivalent.

Proof. Let $f \in L_{\text {loc }}^{1}(\mu)$. We first prove that $\|f\|_{* *} \leq C\|f\|_{*}$. To do so, for any cube $Q$, we set

$$
f_{Q}= \begin{cases}m_{\tilde{Q}} f(x) & \text { if } l(\widetilde{Q}) \leq 1 \\ 0 & \text { otherwise }\end{cases}
$$


Then, if $l(\widetilde{Q}) \leq 1,(2.4)$ holds with $C_{3}=\|f\|_{*}$. If $l(Q) \leq 1$ and $l(\widetilde{Q})>1$, we have

$$
\begin{aligned}
\frac{1}{\mu(\eta Q)} \int_{Q}\left|f(x)-f_{Q}\right| d \mu(x) & =\frac{1}{\mu(\eta Q)} \int_{Q}|f(x)| d \mu(x) \\
& \leq \frac{1}{\mu(\eta Q)} \int_{Q}\left|f(x)-m_{\widetilde{Q}} f\right| d \mu(x)+\frac{\mu(Q)}{\mu(\eta Q)}\left|m_{\tilde{Q}} f\right| \\
& \leq\|f\|_{*}+\frac{\mu(Q)}{\mu(\eta Q)} \frac{\mu(\rho \widetilde{Q})}{\mu(\widetilde{Q})}\|f\|_{*} \leq C\|f\|_{*},
\end{aligned}
$$

since $\widetilde{Q}$ is a $\left(\rho, \beta_{\rho}\right)$-doubling cube. Here we used the fact that $\eta \leq \rho$ in the second step to the last one. Thus, (2.4) holds.

We now verify that for any two cubes $Q \subset R$ with $l(Q) \leq 1$,

$$
\left|f_{Q}-f_{R}\right| \leq C K_{Q, R}\|f\|_{*}
$$

We consider several cases.

Case $1 . l(\widetilde{R}) \geq l(\widetilde{Q})>1$. In this case, (2.7) is obviously true.

Case 2. $l(\widetilde{R})>1 \geq l(\widetilde{Q})$. In this case, we let $R_{0}=\widetilde{4 R}$. By Lemma 1.3, we have $K_{\tilde{Q}, R_{0}} \leq C K_{Q, R}$. From this, it follows that

$$
\begin{aligned}
\left|f_{Q}-f_{R}\right| & =\left|f_{Q}\right|=\left|m_{\tilde{Q}} f\right| \leq\left|m_{\tilde{Q}} f-m_{R_{0}} f\right|+\left|m_{R_{0}} f\right| \\
& \leq\left|m_{\tilde{Q}} f-m_{R_{0}} f\right|+C\|f\|_{*} \leq C K_{Q . R}\|f\|_{*} .
\end{aligned}
$$

Estimate (2.7) also holds.

Case 3. $1 \geq l(\widetilde{R}) \geq l(\widetilde{Q})$. We have $\left|f_{Q}-f_{R}\right|=\left|m_{\tilde{Q}} f-m_{\tilde{R}} f\right|$, and by [13, (2.13)], we know (2.7) is true.

Case 4. $l(\widetilde{R})<l(\widetilde{Q}) \leq 1$. This case is similar to Case 3. By $[13,(2.13)]$ we know (2.7) that holds.

Case 5. $l(\widetilde{R}) \leq 1<l(\widetilde{Q})$. In this case, if we denote $Q_{0}=\widetilde{4 \widetilde{Q}}$, then $l\left(Q_{0}\right)>1$ and by Lemma 1.3 we have

$$
\begin{aligned}
\left|f_{Q}-f_{R}\right| & =\left|f_{R}\right|=\left|m_{\widetilde{R}} f\right| \leq\left|m_{\widetilde{R}} f-m_{Q_{0}} f\right|+\left|m_{Q_{0}} f\right| \\
& \leq C K_{\widetilde{R}, Q_{0}}\|f\|_{*}+\frac{\mu\left(\rho Q_{0}\right)}{\mu\left(Q_{0}\right)} \frac{1}{\mu\left(\eta Q_{0}\right)} \int_{Q_{0}}|f(x)| d \mu(x) \leq C\|f\|_{*} .
\end{aligned}
$$

Here we used the facts that $\eta \leq \rho, K_{\tilde{R}, Q_{0}} \leq C$ by Lemma 1.3 and $Q_{0}$ is a $\left(\rho, \beta_{\rho}\right)$ doubling cube. Thus (2.7) holds.

Case $6.1<l(\widetilde{R})<l(\widetilde{Q})$. In this case $(2.7)$ is trivial.

Thus (2.7) holds in all cases and we obviously have (2.6). So, $\|f\|_{* *} \leq C\|f\|_{*}$.

Let us now prove the converse. We first prove that if $Q$ is a cube with $l(Q)>1$, then

$$
\frac{1}{\mu(\eta Q)} \int_{Q}|f(x)| d \mu(x) \leq C\|f\|_{* *}
$$


In fact, for each $x \in Q \cap \operatorname{supp} \mu$, we choose a cube $Q_{x} \subset \eta Q$ with its center at $x$ and $l\left(Q_{x}\right) \leq 1<v l\left(Q_{x}\right)$, where $v>\max (1,1 /(\eta-1))$. By $(1.1)$, we can take $Q_{x}$ to be a $\left(\rho, \beta_{\rho}\right)$-doubling cube. Then, by the Besicovich covering theorem, we can find a family of points $\left\{x_{i}\right\}_{i} \subset Q \cap \operatorname{supp} \mu$ such that the cubes $\left\{Q_{x_{i}}\right\}_{i}$ form an almost disjoint covering of $Q \cap \operatorname{supp} \mu$. Since $l\left(Q_{x_{i}}\right) \leq 1<v l\left(Q_{x_{i}}\right)$, by (2.5), (2.6) and Lemma 1.3, we have

$$
\left|f_{Q_{x_{i}}}\right| \leq\left|f_{Q_{x_{i}}}-f_{\nu Q_{x_{i}}}\right|+\left|f_{\nu Q_{x_{i}}}\right| \leq C K_{Q_{x_{i}}, v Q_{x_{i}}}\|f\|_{* *}+C\|f\|_{* *} \leq C\|f\|_{* *} .
$$

Since $Q_{x_{i}}$ is $\left(\rho, \beta_{\rho}\right)$-doubling and $\eta \leq \rho$, we also have

$$
\sum_{i} \mu\left(\eta Q_{x_{i}}\right) \leq \sum_{i} \mu\left(\rho Q_{x_{i}}\right) \leq C \sum_{i} \mu\left(Q_{x_{i}}\right) \leq C \mu(\eta Q) .
$$

From (2.9) and (2.10) we deduce

$$
\begin{aligned}
\frac{1}{\mu(\eta Q)} & \int_{Q}|f(x)| d \mu(x) \\
& \leq \frac{1}{\mu(\eta Q)} \sum_{i} \int_{Q_{x_{i}}}\left|f(x)-f_{Q_{x_{i}}}\right| d \mu(x)+\frac{1}{\mu(\eta Q)} \sum_{i} \mu\left(Q_{x_{i}}\right)\left|f_{Q_{x_{i}}}\right| \\
& \leq C \frac{1}{\mu(\eta Q)} \sum_{i} \mu\left(\eta Q_{x_{i}}\right)\|f\|_{* *}+C\|f\|_{* *} \leq C\|f\|_{* *} .
\end{aligned}
$$

Thus (2.8) holds.

Now if $Q$ is a $\left(\rho, \beta_{\rho}\right)$-doubling cube with $l(Q) \leq 1$, by (2.4) and $\eta \leq \rho$, we have

$$
\left|f_{Q}-m_{Q} f\right|=\left|\frac{1}{\mu(Q)} \int_{Q}\left(f-f_{Q}\right) d \mu\right| \leq\|f\|_{* *} \frac{\mu(\eta Q)}{\mu(Q)} \leq C\|f\|_{* *} .
$$

Thus for any cube $Q$ with $l(Q) \leq 1$, by $(2.11)$ when $l(\widetilde{Q}) \leq 1$ or by $(2.6)$ and (2.8) when $l(\widetilde{Q})>1$, we obtain $\left|f_{Q}-m_{\tilde{Q}} f\right| \leq\left|f_{Q}-f_{\tilde{Q}}\right|+\left|f_{\tilde{Q}}-m_{\tilde{Q}} f\right| \leq C\|f\|_{* * *}$. Therefore, if $l(Q) \leq 1$, we have

$$
\begin{aligned}
\frac{1}{\mu(\eta Q)} & \int_{Q}\left|f(x)-m_{\tilde{Q}} f\right| d \mu(x) \\
& \leq \frac{1}{\mu(\eta Q)} \int_{Q}\left|f(x)-f_{Q}\right| d \mu(x)+\frac{1}{\mu(\eta Q)} \int_{Q}\left|f_{Q}-m_{\tilde{Q}} f\right| d \mu(x) \\
& \leq C\|f\|_{* *} .
\end{aligned}
$$

Finally, if $Q \subset R$ with $l(Q) \leq 1$ are $\left(\rho, \beta_{\rho}\right)$-doubling, by $(2.5),(2.11)$ when $l(R) \leq 1$ or by $(2.5),(2.6)$ and $(2.8)$ when $l(R)>1$, we have

$$
\begin{aligned}
\left|m_{Q} f-m_{R} f\right| & \leq\left|m_{Q} f-f_{Q}\right|+\left|f_{Q}-f_{R}\right|+\left|f_{R}-m_{R} f\right| \\
& \leq C\|f\|_{* *}+C K_{Q, R}\|f\|_{* *} \leq C K_{Q, R}\|f\|_{* *} .
\end{aligned}
$$

This proves that $\|f\|_{*} \leq C\|f\|_{* *}$. 
RemarK 2.2. By Proposition 2.3 and Proposition 2.4, we easily see that we obtain equivalent definitions for the space $\operatorname{rbmo}(\mu)$ if instead of cubes centered at points in $\operatorname{supp} \mu$, we consider all the cubes in $\mathbb{R}^{d}$ with sides parallel to the axes. Furthermore, it does not matter if we take balls instead of cubes.

REMARK 2.3. By the proof of (2.7) and the proof of [13, (2.13)], it is easy to see that if $Q \subset R$ with $l(Q) \leq 1$ and $f \in \operatorname{rbmo}(\mu)$, then $\left|m_{\tilde{Q}} f-m_{\tilde{R}} f\right| \leq C K_{Q, R}\|f\|_{*}$.

The following proposition indicates other possible ways of defining $r b m o(\mu)$.

PROPOSITION 2.5. Let $\eta \in(1, \infty), \rho \in[\eta, \infty)$ and $\beta_{\rho}>[\max (\rho, 1 /(\eta-1))]^{n}$ be fixed. For a function $f \in L_{\mathrm{loc}}^{1}(\mu)$, the following are equivalent:

(i) $f \in \operatorname{rbmo}(\mu)$.

(ii) There exists some constant $C_{b} \geq 0$ such that for any cube $Q$ with $l(Q) \leq 1$,

$$
\int_{Q}\left|f-m_{Q} f\right| d \mu \leq C_{b} \mu(\eta Q)
$$

for any two cubes $Q \subset R$ with $l(Q) \leq 1$,

$$
\left|m_{Q} f-m_{R} f\right| \leq C_{b} K_{Q, R}\left[\frac{\mu(\eta Q)}{\mu(Q)}+\frac{\mu(\eta R)}{\mu(R)}\right],
$$

and for any cube $Q$ with $l(Q)>1$,

$$
\int_{Q}|f(x)| d \mu(x) \leq C_{b} \mu(\eta Q) .
$$

(iii) There exists some constant $C_{c} \geq 0$ such that for any $\left(\rho, \beta_{\rho}\right)$-doubling cube $Q$ with $l(Q) \leq 1$,

$$
\int_{Q}\left|f-m_{Q} f\right| d \mu \leq C_{c} \mu(Q),
$$

for any two $\left(\rho, \beta_{\rho}\right)$-doubling cubes $Q \subset R$ with $l(Q) \leq 1$,

$$
\left|m_{Q} f-m_{R} f\right| \leq C_{c} K_{Q, R},
$$

and for any $\left(\rho, \beta_{\rho}\right)$-doubling cube $Q$ with $l(Q)>1$,

$$
\int_{Q}|f(x)| d \mu(x) \leq C_{c} \mu(Q) .
$$

Moreover, the best constants $C_{b}$ and $C_{c}$ are comparable to the rbmo $(\mu)$ norm of $f$. 
PROOF. Let us first prove that (i) implies (ii). If $f \in r b m o(\mu)$, then for any cube $Q$ with $l(Q) \leq 1$, we have

$$
\left|m_{Q} f-m_{\tilde{Q}} f\right| \leq m_{Q}\left(\left|f-m_{\tilde{Q}} f\right|\right) \leq\|f\|_{*} \frac{\mu(\eta Q)}{\mu(Q)} .
$$

From this, we easily follow that (2.12) holds with $C_{b}=2\|f\|_{*}$.

Now let us prove that $(2.13)$ also holds. We consider several cases.

Case 1. $l(\widetilde{Q}) \leq 1$ and $l(\widetilde{R}) \leq 1$. By $(2.18)$ and (2.7) we have

$$
\begin{aligned}
\left|m_{Q} f-m_{R} f\right| & \leq\left|m_{Q} f-m_{\tilde{Q}} f\right|+\left|m_{\tilde{Q}} f-m_{\tilde{R}} f\right|+\left|m_{\tilde{R}} f-m_{R} f\right| \\
& \leq\|f\|_{*} \frac{\mu(\eta Q)}{\mu(Q)}+C K_{Q, R}\|f\|_{*}+\|f\|_{*} \frac{\mu(\eta R)}{\mu(R)} \\
& \leq C K_{Q, R}\left[\frac{\mu(\eta Q)}{\mu(Q)}+\frac{\mu(\eta R)}{\mu(R)}\right]\|f\|_{*},
\end{aligned}
$$

that is, (2.13) holds.

Case 2. $l(\widetilde{Q})>1$ and $l(R) \leq 1<l(\widetilde{R})$. From (2.3) and the facts that $\widetilde{Q}$ and $\widetilde{R}$ are $\left(\rho, \beta_{\rho}\right)$-doubling, we deduce $\left|m_{\tilde{Q}} f-m_{\tilde{R}} f\right| \leq C\|f\|_{*}$. By this and (2.18) we obtain (2.13).

Case 3. $l(\widetilde{Q})>1$ and $l(\widetilde{R}) \leq 1$. In this case, $\widetilde{R} \subset 4 \widetilde{Q}$. Let $Q_{0}=4 \widetilde{4}$. By Lemma 1.3, we have $K_{\tilde{R}, Q_{0}} \leq C$ (see the proof of [13, Lemma 2.8]). By (2.18) and (2.3) we have

$$
\begin{aligned}
\left|m_{\tilde{Q}} f-m_{\tilde{R}} f\right| & \leq C\left|m_{Q_{0}} f-m_{\widetilde{R}} f\right|+\left|m_{Q_{0}} f\right|+\left|m_{\tilde{Q}} f\right| \\
& \leq C K_{\widetilde{R}, Q_{0}}\|f\|_{*}+\left[\frac{\mu\left(\eta Q_{0}\right)}{\mu\left(Q_{0}\right)}+\frac{\mu(\eta \widetilde{Q})}{\mu(\widetilde{Q})}\right]\|f\|_{*} \leq C\|f\|_{*} .
\end{aligned}
$$

We then obtain (2.13) by this and (2.18).

Case 4. $l(\widetilde{Q})>1$ and $1<l(R)<l(\widetilde{R})$. In this case, by (2.3) and (2.18), we have

$$
\begin{aligned}
\left|m_{Q} f-m_{R} f\right| & \leq\left|m_{Q} f-m_{\tilde{Q}} f\right|+\left|m_{\tilde{Q}} f\right|+\left|m_{R} f\right| \\
& \leq\|f\|_{*} \frac{\mu(\eta Q)}{\mu(Q)}+C\|f\|_{*}+\|f\|_{*} \frac{\mu(\eta R)}{\mu(R)} \\
& \leq C K_{Q, R}\left[\frac{\mu(\eta Q)}{\mu(Q)}+\frac{\mu(\eta R)}{\mu(R)}\right]\|f\|_{*} .
\end{aligned}
$$

Thus (2.13) also holds.

Case 5. $l(\widetilde{Q}) \leq 1$ and $l(R) \leq 1<l(\widetilde{R})$. In this case, by [13, (2.14)], we have

$$
\left|m_{\tilde{Q}} f-m_{\tilde{R}} f\right| \leq C K_{Q, R}\|f\|_{*} .
$$

From this and (2.18), we then easily deduce (2.13). 
Case 6. $l(\widetilde{Q}) \leq 1$ and $l<l(R) \leq l(\widetilde{R})$. In this case, by the proof of [13, (2.14)], we still have (2.19) holds. We then, by (2.19), (2.18) and (2.3), obtain that

$$
\begin{aligned}
\left|m_{Q} f-m_{R} f\right| & \leq\left|m_{Q} f-m_{\tilde{Q}} f\right|+\left|m_{\tilde{Q}} f-m_{\tilde{R}} f\right|+\left|m_{\tilde{R}} f\right|+\left|m_{R} f\right| \\
& \leq\|f\|_{*} \frac{\mu(\eta Q)}{\mu(Q)}+C K_{Q, R}\|f\|_{*}+C\|f\|_{*}+\|f\|_{*} \frac{\mu(\eta R)}{\mu(R)} \\
& \leq C K_{Q, R}\left[\frac{\mu(\eta Q)}{\mu(Q)}+\frac{\mu(\eta R)}{\mu(R)}\right]\|f\|_{*} .
\end{aligned}
$$

Thus, in all cases, (2.13) holds. Estimate (2.14) is obvious.

That (ii) implies (iii) is trivial.

Let us now see that (iii) implies (i). We first prove (2.3). Let $Q$ be a cube with $l(Q)>1$. We consider the same covering as in the proof of (2.8). Since $l\left(v \widetilde{Q_{x_{i}}}\right) \geq l\left(v Q_{x_{i}}\right)>1$, by $(2.16)-(2.17)$ and Lemma 1.3 , we then have

$$
\begin{aligned}
\left|m_{Q_{x_{i}}} f\right| & \leq\left|m_{Q_{x_{i}}} f-m_{\nu \widetilde{Q}_{x_{i}}} f\right|+\left|m_{\nu \widetilde{Q}_{x_{i}}} f\right| \leq C_{c} K_{Q_{x_{i}}, \nu \widetilde{Q}_{x_{i}}}+C_{c} \\
& \leq C_{c}\left[K_{Q_{x_{i}}, \nu Q_{x_{i}}}+K_{\nu Q_{x_{i}}, \nu \widetilde{Q}_{x_{i}}}\right]+C_{c} \leq C C_{c} .
\end{aligned}
$$

From this, (2.15) and (2.10), we deduce

$$
\begin{aligned}
\frac{1}{\mu(\eta Q)} \int_{Q}|f(x)| d \mu(x) \leq & \frac{1}{\mu(\eta Q)} \sum_{i} \int_{Q_{x_{i}}}|f(x)| d \mu(x) \\
\leq & \frac{1}{\mu(\eta Q)} \sum_{i} \int_{Q_{x_{i}}}\left|f(x)-m_{Q_{x_{i}}} f\right| d \mu(x) \\
& +\frac{1}{\mu(\eta Q)} \sum_{i}\left|m_{Q_{x_{i}}} f\right| \mu\left(Q_{x_{i}}\right) \\
\leq & \frac{C_{c}}{\mu(\eta Q)} \sum_{i} \mu\left(Q_{x_{i}}\right) \leq C C_{c} .
\end{aligned}
$$

Thus (2.3) holds.

Finally, we need to prove that (2.15) holds for any cube $Q$ with $l(Q) \leq 1$. We suppose $Q$ is not a $\left(\rho, \beta_{\rho}\right)$-doubling cube. The argument is similar to the one given in the proof of $\left[13\right.$, Lemma 2.10]. Since $\beta_{\rho}>[\max (\rho, 1 /(\eta-1))]^{n}$, we can choose $v>\max (\rho, 1 /(\eta-1))$ such that $\beta_{\rho}>\nu^{n}$. We know that for all $x \in Q \cap \operatorname{supp} \mu$, there exists some $\left(\rho, \beta_{\rho}\right)$-doubling cube centered at $x$ with side length $\nu^{-k} l(Q)$, where $k \in \mathbb{N}$. We denote by $Q_{x}$ the biggest cube satisfying these properties. Since $\beta_{\rho}>v^{n}$, we then can prove, by Lemma 1.3 , that $K_{Q_{x}, \tilde{Q}} \leq C$. Notice that $Q_{x} \subset \eta Q \subset \widetilde{Q}$. By (2.16), we have

$$
\left|m_{Q_{x}} f-m_{\tilde{Q}} f\right| \leq C C_{c} .
$$


By Besicovich's covering theorem, there are points $x_{i} \in Q$ such that $Q \cap \operatorname{supp} \mu$ is covered by a family of cubes $\left\{Q_{x_{i}}\right\}_{i}$ with bounded overlap. From this and (2.20), we deduce

$$
\begin{aligned}
\int_{Q}\left|f-m_{\tilde{Q}} f\right| d \mu & \leq \sum_{i} \int_{Q_{x_{i}}}\left|f-m_{\tilde{Q}} f\right| d \mu \\
& \leq \sum_{i} \int_{Q_{x_{i}}}\left|f-m_{Q_{x_{i}}} f\right| d \mu+\sum_{i}\left|m_{\tilde{Q}} f-m_{Q_{x_{i}}} f\right| \mu\left(Q_{x_{i}}\right) \\
& \leq C C_{c} \mu(\eta Q) .
\end{aligned}
$$

Thus $f \in \operatorname{rbmo}(\mu)$.

The following theorem is a version of John-Nirenberg's inequality related to the space $r b m o(\mu)$. To prove it we adapt the arguments of [13, Theorem 3.1] which is, in fact, the John-Nirenberg's inequality for the functions in $R B M O(v)$ (see also [7]).

THEOREM 2.6. Let $f \in \operatorname{rbmo}(\mu)$ and let $\eta \in(1, \infty), \rho \in[\eta, \infty)$ and $\left\{f_{Q}\right\}_{Q}$ be a collection of numbers satisfying

$$
\begin{gathered}
\sup _{Q: l(Q) \leq 1} \frac{1}{\mu(2 Q)} \int_{Q}\left|f(x)-f_{Q}\right| d \mu(x) \leq C\|f\|_{*}, \\
\left|f_{Q}-f_{R}\right| \leq C K_{Q, R}\|f\|_{*}
\end{gathered}
$$

for any two cubes $Q \subset R$ with $l(Q) \leq 1$, and for any cube $Q$ with $l(Q)>1$,

$$
\left|f_{Q}\right| \leq C\|f\|_{*} \text {. }
$$

Then, for any cube $Q$ with $l(Q) \leq 1$ and any $\lambda>0$, we have

$$
\mu\left\{x \in Q:\left|f(x)-f_{Q}\right|>\lambda\right\} \leq C_{4} \mu(\eta Q) \exp \left(-\frac{C_{5} \lambda}{\|f\|_{*}}\right),
$$

and for any cube $Q$ with $l(Q)>1$ and any $\lambda>0$, we have

$$
\mu\{x \in Q:|f(x)|>\lambda\} \leq C_{4} \mu(\eta Q) \exp \left(-\frac{C_{5} \lambda}{\|f\|_{*}}\right) .
$$

Here $C_{4} \geq 0$ and $C_{5} \geq 0$ depend on $\eta$ and $\rho$ and are independent of $\lambda$ and $f$.

To prove our theorem, we need the following lemma.

LEMMA 2.7. Let $f \in \operatorname{rbmo}(\mu)$ and let $\left\{f_{Q}\right\}_{Q}$ be a collection of numbers satisfying (2.21)-(2.23). If $Q$ and $R$ are cubes such that $l(Q) \sim l(R)$ and $\operatorname{dist}(Q, R) \leq l(Q)$, then

$$
\left|f_{Q}-f_{R}\right| \leq C\|f\|_{*}
$$


Proof. If $\min (l(Q), l(R))>1$, then we obtain (2.26) by (2.23).

If $\min (l(Q), l(R)) \leq 1$, let $R^{\prime}$ be the smallest cube concentric with $R$ containing $Q$ and $R$. Since $l(Q) \sim l\left(R^{\prime}\right) \sim l(R)$, we have $K_{Q, R^{\prime}} \leq C$ and $K_{R, R^{\prime}} \leq C$. If $\max (l(Q), l(R)) \leq 1$, by $(2.22)$, we then have

$$
\left|f_{Q}-f_{R}\right| \leq\left|f_{Q}-f_{R^{\prime}}\right|+\left|f_{R^{\prime}}-f_{R}\right| \leq C\left(K_{Q, R^{\prime}}+K_{R, R^{\prime}}\right)\|f\|_{*} \leq C\|f\|_{*} .
$$

If $\max (l(Q), l(R))>1$, say, $l(R)>1$, then $l(Q) \leq 1$ and $l\left(R^{\prime}\right)>1$. Thus, by (2.22) and (2.23), we obtain

$$
\left|f_{Q}-f_{R}\right| \leq\left|f_{Q}-f_{R^{\prime}}\right|+\left|f_{R^{\prime}}\right|+\left|f_{R}\right| \leq C K_{Q, R^{\prime}}\|f\|_{*}+C\|f\|_{*} \leq C\|f\|_{*} .
$$

Thus (2.26) also holds. If $l(R) \leq 1$, then $l(Q)>1$ and $l\left(R^{\prime}\right)>1$, we then can obtain (2.26) also by (2.22) and (2.23).

The following lemma is similar to [13, Lemma 3.3] and its proof is also similar, by replacing [13, Proposition 2.5] by Proposition 2.2, to the proof of [13, Lemma 3.3]. We omit the details.

LEMMA 2.8. Let $f \in \operatorname{rbmo}(\mu)$. Given $q>0$, we let

$$
f_{q}(x)= \begin{cases}f(x) & \text { if }|f(x)| \leq q ; \\ q f(x) /|f(x)| & \text { if }|f(x)|>q .\end{cases}
$$

Then $f_{q} \in \operatorname{rbmo}(\mu)$ with $\left\|f_{q}\right\| \leq C\|f\|_{*}$.

We also have a remark similar to [13, Remark 3.4], which will be used in the proof below.

REMARK 2.4. Let $f \in r b m o(\mu)$ and let $\left\{f_{Q}\right\}_{Q}$ be a collection of numbers satisfying (2.21)-(2.23). We set $f_{Q,+}=\max \left(f_{Q}, 0\right)$ and $f_{Q,-}=-\min \left(f_{Q}, 0\right)$ and we set

$$
f_{q, Q}=\min \left(f_{Q,+}, q\right)-\min \left(f_{Q,-}, q\right) .
$$

Then, it is easily seen that

$$
\begin{gathered}
\sup _{Q: \ell(Q) \leq 1} \frac{1}{\mu(2 Q)} \int_{Q}\left|f_{q}(x)-f_{q, Q}\right| d \mu(x) \leq C\|f\|_{*}, \\
\left|f_{q, Q}-f_{q, R}\right| \leq C K_{Q, R}\|f\|_{*}
\end{gathered}
$$

for any two cubes $Q \subset R$ with $l(Q) \leq 1$, and for any cube $Q$ with $l(Q)>1$,

$$
\left|f_{q, Q}\right| \leq C\|f\|_{*},
$$

where $C \geq 0$ is independent of $q$. 
PROOF OF THEOREM 2.6. We prove (2.24) and (2.25) for $\eta=\rho=2$. The proof for other values of $\eta$ and $\rho$ is similar; see also Remark 2.1.

Let $f \in \operatorname{rbmo}(\mu)$ and assume first that $f$ is bounded. Let $Q_{0}$ be some fixed cube in $\mathbb{R}^{d}$ with $l\left(Q_{0}\right)>1$ and we write $Q_{0}^{\prime}=\frac{3}{2} Q_{0}$. Let $B$ be some positive constant which will be fixed later. By [13, Remark 2.3], for $\mu$-almost any $x \in Q_{0}$ such that $|f(x)|>B\|f\|_{*}$, there exists some doubling cube $Q_{x}$ centered at $x$ satisfying

$$
m_{Q_{x}}|f|>B\|f\|_{*} .
$$

Moreover, we may assume that $Q_{x}$ is the biggest doubling cube satisfying (2.27) with side length $2^{-k} l\left(Q_{0}\right)$ for some integer $k \geq 0$ and $l\left(Q_{x}\right) \leq \frac{1}{10} l\left(Q_{0}\right)$. By Besicovich's covering theorem, there exists an almost disjoint subfamily $\left\{Q_{i}\right\}_{i}$ such that

$$
\left\{x \in Q_{0}:|f(x)|>B\|f\|_{*}\right\} \subset \bigcup_{i} Q_{i} .
$$

By Proposition 2.3 and Proposition 2.4, we deduce from (2.21)-(2.23) that

$$
\frac{1}{\mu(4 R / 3)} \int_{R}|f(x)| d \mu(x) \leq C\|f\|_{*}
$$

for any cube $R$ with $l(R)>1$. Thus, if we choose $B$ big enough, by (2.29), we then have

$$
\begin{aligned}
\sum_{i} \mu\left(Q_{i}\right) & \leq \sum_{i} \frac{1}{B\|f\|_{*}} \int_{Q_{i}}|f(x)| d \mu(x) \leq \frac{1}{B\|f\|_{*}} \int_{Q_{0}^{\prime}}|f(x)| d \mu(x) \\
& \leq C \frac{\mu\left(\frac{4}{3} Q_{0}^{\prime}\right)}{B} \leq C \frac{\mu\left(2 Q_{0}\right)}{B} \leq \frac{\mu\left(2 Q_{0}\right)}{4 \beta_{\rho}} .
\end{aligned}
$$

We now prove that

$$
\left|f_{Q_{i}}\right| \leq C_{6}\|f\|_{*}
$$

If $l\left(Q_{i}\right)>1,(2.31)$ is true by $(2.23)$. If $l\left(Q_{i}\right) \leq 1$, we consider the cube $\widetilde{2 Q_{i}}$ and we then consider several cases.

If $l\left(\widetilde{2 Q_{i}}\right)>10 l\left(Q_{0}\right)$, then there exists some cube $2^{m} Q_{i}, m \geq 1$, containing $Q_{0}$ and such that $l\left(Q_{0}\right) \sim l\left(2^{m} Q_{i}\right) \leq l\left(\widetilde{2 Q_{i}}\right)$. Thus, if $l\left(2 Q_{i}\right)>1$, then

$$
\left|f_{Q_{i}}\right| \leq\left|f_{Q_{i}}-f_{2 Q_{i}}\right|+\left|f_{2 Q_{i}}\right| \leq C K_{Q_{i}, 2 Q_{i}}\|f\|_{*}+C\|f\|_{*} \leq C\|f\|_{*} .
$$

If $l\left(2 Q_{i}\right) \leq 1<l\left(2^{m} Q_{i}\right)$, then

$$
\begin{aligned}
\left|f_{Q_{i}}\right| & \leq\left|f_{Q_{i}}-f_{2 Q_{i}}\right|+\left|f_{2 Q_{i}}-f_{2^{m} Q_{i}}\right|+\left|f_{2^{m} Q_{i}}\right| \\
& \leq C K_{Q_{i}, 2 Q_{i}}\|f\|_{*}+C K_{2 Q_{i}, 2^{m} Q_{i}}\|f\|_{*}+C\|f\|_{*} \leq C\|f\|_{*},
\end{aligned}
$$


where we used (2.23) and the fact that $K_{2 Q_{i}, 2^{m} Q_{i}} \leq C$ since there are no doubling cubes of the form $2^{k} Q_{i}$ between $Q_{i}$ and $2^{m} Q_{i}$ and $K_{2 Q_{i}, 2^{m} Q_{i}} \leq C K_{Q_{i}, 2^{m} Q_{i}} \leq C$ by Lemma 1.3. If $l\left(2^{m} Q_{i}\right) \leq 1<l\left(Q_{0}\right)$, we have

$$
\begin{aligned}
\left|f_{Q_{i}}\right| & \leq\left|f_{Q_{i}}-f_{2 Q_{i}}\right|+\left|f_{2 Q_{i}}-f_{2^{m} Q_{i}}\right|+\left|f_{2^{m} Q_{i}}-f_{Q_{0}}\right|+\left|f_{Q_{0}}\right| \\
& \leq C\left[K_{Q_{i}, 2 Q_{i}}+K_{2 Q_{i}, 2^{m} Q_{i}}+K_{2^{m} Q_{i}, Q_{0}}\right]\|f\|_{*}+C\|f\|_{*} \leq C\|f\|_{*}
\end{aligned}
$$

by (2.23) and Lemma 1.3 again. Thus, in this case, (2.31) is true.

Now if $1<l\left(\widetilde{2 Q_{i}}\right) \leq 10 l\left(Q_{0}\right)$, then by $(2.22)-(2.23)$, and Lemma 1.3 , we have

$$
\begin{aligned}
\left|f_{Q_{i}}\right| & \leq\left|f_{Q_{i}}-f_{\widetilde{2 \widetilde{Q}_{i}}}\right|+\left|f_{\widetilde{2 Q_{i}}}\right| \leq C K_{Q_{i}, \widetilde{Q_{i}}}\|f\|_{*}+C\|f\|_{*} \\
& \leq C\left[K_{Q_{i}, 2 Q_{i}}+K_{2 Q_{i}, 2 \widetilde{Q}_{i}}\right]\|f\|_{*}+C\|f\|_{*} \leq C\|f\|_{*} .
\end{aligned}
$$

If $l\left(Q_{0}\right) / 10<l\left(\widetilde{2 Q_{i}}\right) \leq 1$, then from (2.22)-(2.23), Lemma 2.7 and Lemma 1.3 we deduce

$$
\begin{aligned}
\left|f_{Q_{i}}\right| & \leq\left|f_{Q_{i}}-f_{2 \widetilde{Q_{i}}}\right|+\left|f_{\widetilde{2 Q_{i}}}-f_{Q_{0}}\right|+\left|f_{Q_{0}}\right| \\
& \leq C\left[K_{Q_{i}, \widetilde{2 Q_{i}}}+K_{2 \widetilde{Q}_{i}, Q_{0}}\right]\|f\|_{*}+C\|f\|_{*} \leq C\|f\|_{*} .
\end{aligned}
$$

If $l\left(\widetilde{2 Q_{i}}\right) \leq l\left(Q_{0}\right) / 10$, then, by the maximality of $Q_{i}$, we have $m_{2 \widetilde{Q}_{i}}(|f|) \leq B\|f\|_{*}$ which implies that

$$
\left|m_{2 \widetilde{Q}_{i}}(f)\right| \leq B\|f\|_{*} .
$$

If $l\left(\widetilde{2 Q_{i}}\right)>1$, then $(2.32)$ indicates (2.31). If $l\left(\widetilde{2 Q_{i}}\right) \leq 1$, by $(2.21),(2.33)$ and Lemma 1.3 we have

$$
\begin{aligned}
\left|f_{Q_{i}}\right| & \leq\left|f_{Q_{i}}-f_{\widetilde{2 \widetilde{Q}_{i}}}\right|+\left|f_{\widetilde{2 \widetilde{Q}_{i}}}-m_{\widetilde{2 Q_{i}}}(f)\right|+\left|m_{\widetilde{2 Q_{i}}}(f)\right| \\
& \leq C K_{Q_{i}, \widetilde{2 Q_{i}}}\|f\|_{*}+B\|f\|_{*}+\frac{1}{\mu\left(\widetilde{\left.2 Q_{i}\right)}\right.} \int_{\widetilde{2 Q_{i}}}\left|f(x)-f_{\widetilde{2} \widetilde{Q}_{i}}\right| d \mu(x) \\
& \leq C\|f\|_{*}+C \frac{\mu\left(2 \widetilde{2} \widetilde{Q}_{i}\right)}{\mu\left(\widetilde{2 Q_{i}}\right)}\|f\|_{*} \\
& \leq C\|f\|_{*} .
\end{aligned}
$$

Thus, in any case, (2.31) holds.

Now we consider the function

$$
\begin{aligned}
X(t)= & \sup _{Q: \ell(Q) \leq 1} \frac{1}{\mu(2 Q)} \int_{Q} \exp \left(\left|f(x)-f_{Q}\right| \frac{t}{\|f\|_{*}}\right) d \mu(x) \\
& +\sup _{Q: \nVdash(Q)>1} \frac{1}{\mu(2 Q)} \int_{Q} \exp \left(|f(x)| \frac{t}{\|f\|_{*}}\right) d \mu(x) .
\end{aligned}
$$


Since $f$ is bounded, $X(t)<\infty$. By (2.28), (2.30) and (2.31), we have

$$
\begin{aligned}
\frac{1}{\mu\left(2 Q_{0}\right)} & \int_{Q_{0}} \exp \left(|f(x)| \frac{t}{\|f\|_{*}}\right) d \mu(x) \\
\leq & \frac{1}{\mu\left(2 Q_{0}\right)} \int_{Q_{0} \backslash \cup_{i} Q_{i}} \exp (B t) d \mu(x) \\
& +\frac{1}{\mu\left(2 Q_{0}\right)} \sum_{i, l\left(Q_{i}\right) \leq 1} \int_{Q_{i}} \exp \left(\left|f(x)-f_{Q_{i}}\right| \frac{t}{\|f\|_{*}}\right) d \mu(x) \exp \left(C_{6} t\right) \\
& +\frac{1}{\mu\left(2 Q_{0}\right)} \sum_{i, l\left(Q_{i}\right)>1} \int_{Q_{i}} \exp \left(|f(x)| \frac{t}{\|f\|_{*}}\right) d \mu(x) \\
\leq & \exp (B t)+\frac{1}{4} X(t) \exp \left(C_{6} t\right)+\frac{1}{4} X(t) .
\end{aligned}
$$

If $l\left(Q_{0}\right) \leq 1$, by a modification of the argument in [13, pages 108-110], we can prove

$$
\frac{1}{\mu\left(2 Q_{0}\right)} \int_{Q_{0}} \exp \left(\left|f(x)-f_{Q_{0}}\right| \frac{t}{\|f\|_{*}}\right) d \mu(x) \leq \exp (B t)+\frac{1}{4} X(t) \exp \left(C_{6} t\right) .
$$

From (2.34) and (2.35), it follows that

$$
X(t)\left[\frac{3}{4}-\frac{1}{2} \exp \left(C_{6} t\right)\right] \leq 2 \exp (B t)
$$

Thus, for $t_{0}$ small enough, we have $X\left(t_{0}\right) \leq C_{7}$, where $C_{7} \geq 0$ depends on $t_{0}, B$ and $C_{6}$.

Therefore, if $f$ is bounded and $l(Q) \leq 1$, then

$$
\begin{aligned}
\mu\left\{x \in Q:\left|f(x)-f_{Q}\right|>\lambda\|f\|_{*} / t_{0}\right\} & \leq \int_{Q} \exp \left(\frac{t_{0}\left|f(x)-f_{Q}\right|}{\|f\|_{*}}\right) \exp (-\lambda) d \mu(x) \\
& \leq C_{7} \mu(2 Q) \exp (-\lambda)
\end{aligned}
$$

which is equivalent to (2.24).

If $f$ is bounded and $l(Q)>1$, then

$$
\begin{aligned}
\mu\left\{x \in Q:|f(x)|>\lambda\|f\|_{*} / t_{0}\right\} & \leq \int_{Q} \exp \left(\frac{t_{0}|f(x)|}{\|f\|_{*}}\right) \exp (-\lambda) d \mu(x) \\
& \leq C_{7} \mu(2 Q) \exp (-\lambda),
\end{aligned}
$$

which is equivalent to (2.25).

When $f$ is not bounded, by Lemma 2.8 , Remark 2.3 , and a similar argument to that in [13, page 111], we can prove that (2.24) and (2.25) are also true. 
From Theorem 2.6, we can easily deduce that the following spaces $r b m o^{p}(\mu)$ coincide for all $p \in[1, \infty)$. Given $\eta \in(1, \infty), \rho \in[\eta, \infty)$ and $p \in[1, \infty)$, we say that $f \in L_{\text {loc }}^{1}(\mu)$ is in $r b m o^{p}(\mu)$ if there exists some constant $C_{8} \geq 0$ such that for any cube $Q$ centered at some point of $\operatorname{supp} \mu$ with $l(Q) \leq 1$,

$$
\begin{gathered}
\left\{\frac{1}{\mu(\eta Q)} \int_{Q}\left|f-m_{\tilde{Q}} f\right|^{p} d \mu\right\}^{1 / p} \leq C_{8}, \\
\left|m_{Q} f-m_{R}\right| \leq C_{8} K_{Q, R}
\end{gathered}
$$

for any two $\left(\rho, \beta_{\rho}\right)$-doubling cubes $Q \subset R$ with $l(Q) \leq 1$, and for any cube $Q$ with $l(Q)>1$,

$$
\left\{\frac{1}{\mu(\eta Q)} \int_{Q}|f(x)|^{p} d \mu\right\}^{1 / p} \leq C_{8} .
$$

Moreover, we define the minimal constant $C_{8}$ as the $r b m o^{p}(\mu)$ norm of $f$ and we denote it by $\|f\|_{*, p}$.

REMARK 2.5. Arguing as for $p=1$, one can prove that another equivalent definition for $r b m o^{p}(\mu)$ can be given in terms of the numbers $\left\{f_{Q}\right\}_{Q}$ as in (2.4)-(2.6). Moreover, Proposition 2.5 is also true for $r b m o^{p}(\mu)$ with any $p \in(1, \infty)$. We omit the details.

By using Theorem 2.6, we can prove the following corollary. See the proof of [13, Corollary 3.5 ] for the details.

COROLlaRY 2.9. For $p \in[1, \infty)$, the spaces $r b m o^{p}(\mu)$ coincide and their norms $\|\cdot\|_{*, p}$ are equivalent.

Finally let us give another useful property of the space $r b m o(\mu)$. To do so, given a cube $Q \subset \mathbb{R}^{d}$ and $f \in L_{\text {loc }}^{1}(\mu)$, we let $\alpha_{Q}(f)$ be the constant for which $\inf _{\alpha} m_{Q}(|f-\alpha|)$ is obtained. It is known that the constant $\alpha_{Q}(f)$, which may be not unique, satisfies

$$
\mu\left\{x \in Q: f(x)>\alpha_{Q}(f)\right\} \leq \mu(Q) / 2
$$

and

$$
\mu\left\{x \in Q: f(x)<\alpha_{Q}(f)\right\} \leq \mu(Q) / 2 ;
$$

see [7, page 30] and [13, pages 115-116].

Given $\eta \in(1, \infty), \rho \in[\eta, \infty), \beta_{\rho}$ big enough and $f \in L_{\text {loc }}^{1}(\mu)$, we denote by

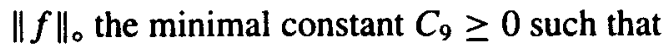

$$
\sum_{Q: I(Q) \leq 1} \frac{1}{\mu(\eta Q)} \int_{Q}\left|f-\alpha_{\tilde{Q}}(f)\right| d \mu \leq C_{9},
$$


for any two $\left(\rho, \beta_{\rho}\right)$-doubling cube $Q \subset R$ with $l(Q) \leq 1$,

$$
\left|\alpha_{Q}(f)-\alpha_{R}(f)\right| \leq C_{9} K_{Q, R},
$$

and for any $\left(\rho, \beta_{\rho}\right)$-doubling cube $Q$ with $l(Q)>1$,

$$
\left|\alpha_{Q}(f)\right| \leq C_{9} .
$$

Then we have the following proposition which is similar to [13, Lemma 4.5].

PROPOSITION 2.10. $\|\cdot\|_{0}$ is a norm which is equivalent to $\|\cdot\|_{*}$.

PROOF. Let us first prove that $\|f\|_{* *} \leq C\|f\|_{0}$. To this end, for any cube $Q \subset \mathbb{R}^{d}$, we define

$$
f_{Q}= \begin{cases}\alpha_{\tilde{Q}}(f) & \text { if } l(\widetilde{Q}) \leq 1 ; \\ 0 & \text { otherwise }\end{cases}
$$

To prove our claim we only need to prove that if $Q \subset R$ with $l(Q) \leq 1$,

$$
\left|f_{Q}-f_{R}\right| \leq C\|f\|_{\circ} K_{Q, R} .
$$

But, this can be proved by a similar way to (2.7) and we omit the details.

Now, let us see the converse. We want to prove that $\|f\|_{0} \leq C\|f\|_{* *}$. Let $Q$ be any $\left(\rho, \beta_{\rho}\right)$-doubling cube with $l(Q)>1$. Then, by the definition of $\alpha_{Q}(f)$, we have

$$
\left|\alpha_{Q}(f) \mu(Q)-\int_{Q} f d \mu(x)\right| \leq \int_{Q}\left|f-\alpha_{Q}(f)\right| d \mu \leq \int_{Q}|f| d \mu .
$$

From this, it follows that

$$
\left|\alpha_{Q}(f)\right| \mu(Q) \leq 2 \int_{Q}|f| d \mu
$$

By (2.8) we then have

$$
\left|\alpha_{Q}(f)\right| \leq C\|f\|_{* *},
$$

that is, (2.40) holds.

To verify (2.38), we first see that if $Q$ is a $\left(\rho, \beta_{\rho}\right)$-doubling cube with $l(Q) \leq 1$, then

$$
\begin{aligned}
\left|f_{Q}-\alpha_{Q}(f)\right|= & \left|\frac{1}{\mu(Q)} \int_{Q}\left[f_{Q}-\alpha_{Q}(f)\right] d \mu\right| \\
\leq & \frac{1}{\mu(Q)} \int_{Q}\left|f_{Q}-f(x)\right| d \mu+ \\
& \frac{1}{\mu(Q)} \int_{Q}\left|f(x)-\alpha_{Q}(f)\right| d \mu \\
\leq & \frac{2}{\mu(Q)} \int_{Q}\left|f(x)-\alpha_{Q}(f)\right| d \mu \leq C\|f\|_{* *} .
\end{aligned}
$$


Now, replacing (2.8) and (2.11) in the last part of the proof of Proposition 2.4, respectively, by (2.42) and (2.43), we then can prove that (2.38) and (2.39) also hold with $C_{9} \leq C\|f\|_{* *}$. Thus our claim holds.

\section{The space $h_{a t b}^{1, p}(\mu)$ with $1<p \leq \infty$}

We first introduce the space $h_{\text {atb }}^{1, \infty}(\mu)$ and present some properties of this space. In the following, for a fixed $\rho \in(1, \infty)$, a function $b \in L_{\text {loc }}^{1}(\mu)$ is called an atomic block if it satisfies

(i) there exists some cube $R$ such that $\operatorname{supp} b \subset R$,

(ii) $\int_{R} b(x) d \mu(x)=0$,

(iii) there are functions $a_{j}$ supported on cubes $Q_{j} \subset R$ and numbers $\lambda_{j} \in \mathbb{R}$ such that $b=\sum_{j=1}^{\infty} \lambda_{j} a_{j}$ and $\|a\|_{L^{\infty}(\mu)} \leq\left[\mu\left(\rho Q_{j}\right) K_{Q_{j}, R}\right]^{-1}$.

Then we define $|b|_{h_{a b}^{1, \infty}(\mu)}=\sum_{j}\left|\lambda_{j}\right|$. A function $b \in L_{\text {loc }}^{1}(\mu)$ is called $a$ block if it satisfies only (i) and (iii).

Moreover, we say that $f \in h_{a t b}^{1, \infty}(\mu)$ if there are atomic blocks or blocks $b_{i}$ such that

$$
f=\sum_{i} b_{i}
$$

where $\sum_{i}\left|b_{i}\right|_{h_{a i b}^{1, \infty}(\mu)}^{\alpha^{\prime}}<\infty, b_{i}$ is an atomic block if supp $b_{i} \subset R_{i}$ and $l\left(R_{i}\right) \leq 1$, and $b_{i}$ is a block if $\operatorname{supp} b_{i} \subset R_{i}$ and $l\left(R_{i}\right)>1$. And we define the $h_{a t b}^{1, \infty}(\mu)$ norm of $f$ by letting $\|f\|_{h_{a b i}^{1, \infty}(\mu)}=\inf \sum_{i}\left|b_{i}\right|_{h_{a b}^{1, \infty}(\mu)}$, where the infimum is taken over all possible decompositions of $f$ in atomic blocks or blocks.

We remark that the atomic blocks were first introduced by Tolsa in [13]. He used them to define the Hardy space $H_{a t b}^{1, \infty}(\mu)$. To be precise, the Hardy space $H_{a, b}^{1, \infty}(\mu)$ consists of all $L^{1}(\mu)$ functions which can be represented as in (3.1) with all the $b_{i}$ 's being atomic blocks. Obviously, $H_{a t b}^{1, \infty}(\mu) \subsetneq h_{a t b}^{1, \infty}(\mu) \subsetneq L^{1}(\mu)$.

On this local Hardy space, we have the following basic properties. The proof of Proposition 3.1 is similar to the proof of [13, Proposition 4.1]. We omit the details here.

PROPOSITION 3.1. $\quad$ (i) The space $h_{a t b}^{1, \infty}(\mu)$ is a Banach space.

(ii) $h_{a t b}^{1, \infty}(\mu) \subset L^{1}(\mu)$ with $\|f\|_{L^{\prime}(\mu)} \leq\|f\|_{h_{a t b}^{1, \infty}(\mu)}$.

(iii) The definition of $h_{a t b}^{1, \infty}(\mu)$ is independent of the choice of the constant $\rho>1$.

The spaces $h_{a t b}^{1, \infty}(\mu)$ and $r b m o(\mu)$ are closely related. In fact, we prove that the dual of $h_{a t b}^{1, \infty}(\mu)$ is $r b m o(\mu)$. We first have the following inclusion. 
LEMMA 3.2. Let $g \in \operatorname{rbmo}(\mu)$. Then the linear functional $L_{g}(f)=\int_{\mathbb{R}^{d}} f g d \mu$ defined on bounded functions $f$ with compact supports extends to a continuous linear functional $L_{g}$ on $h_{a t b}^{1, \infty}(\mu)$ with $\left\|L_{g}\right\|_{h_{a t b}^{1, \infty}(\mu)^{*}} \leq C\|g\|_{*}$. That is, $r b m o(\mu) \subset h_{a t b}^{1, \infty}(\mu)^{*}$.

Proof. Following some standard arguments (see, for example, [3, pages 294 296]), we only need to prove that if $b$ is an atomic block with supp $b \subset R$ and $l(R) \leq 1$ or a block with supp $b \subset R$ and $l(R)>1$ and $g \in \operatorname{rbmo}(\mu)$, then

$$
\left|\int_{\mathbb{R}^{d}} b g d \mu\right| \leq C|b|_{h_{a b b}^{1, \infty}(\mu)}\|g\|_{*} \text {. }
$$

If $b$ is an atomic block, then [13, Lemma 4.3] can be adopted to show (3.2). We now prove (3.2) when $b$ is a block with supp $b \subset R$ and $l(R)>1$. Suppose $b=\sum_{i} \lambda_{i} a_{i}$ and let $\left\{g_{Q}\right\}_{Q}$ be the collection of the numbers satisfying (2.4)-(2.6) of the definition of $g \in \operatorname{rbmo}(\mu)$. We then write

$$
\left|\int_{\mathbb{R}^{d}} b g d \mu\right| \leq\left|\int_{R} b\left(g-g_{R}\right) d \mu\right|+\left|g_{R}\right| \int_{R}|b| d \mu .
$$

By (2.6) and the definition of $b$, we have

$$
\begin{aligned}
\left|g_{R}\right| \int_{R}|b| d \mu & \leq C\|g\|_{*} \sum_{i}\left|\lambda_{i}\right| \int_{Q_{i}}\left|a_{j}\right| d \mu \\
& \leq C\|g\|_{*} \sum_{i}\left|\lambda_{i}\right| \frac{\mu\left(Q_{i}\right)}{\mu\left(\rho Q_{i}\right) K_{Q_{i}, R}} \\
& \leq C\|g\|_{*} \sum_{i}\left|\lambda_{i}\right| \leq C\|g\|_{*}|b|_{h_{a b}^{1, \infty}(\mu)} .
\end{aligned}
$$

Moreover, if $l\left(Q_{i}\right)>1$, by (2.6) and (2.8), we obtain

$$
\begin{aligned}
\int_{Q_{i}}\left|g-g_{R}\right| d \mu & \leq \int_{Q_{i}}|g| d \mu+\left|g_{R}\right| \mu\left(Q_{i}\right) \\
& \leq C\left[\mu\left(\rho Q_{i}\right)+\mu\left(Q_{i}\right)\right]\|g\|_{*} \leq C \mu\left(\rho Q_{i}\right)\|g\|_{*} .
\end{aligned}
$$

If $l\left(Q_{i}\right) \leq 1$, by $(2.4)$ and $(2.5)$, we then have

$$
\begin{aligned}
\int_{Q_{i}}\left|g-g_{R}\right| d \mu & \leq \int_{Q_{i}}\left|g-g_{Q_{i}}\right| d \mu+\left|g_{Q_{i}}-g_{R}\right| \mu\left(Q_{i}\right) \\
& \leq C\left[\mu\left(\rho Q_{i}\right)+K_{Q_{i}, R} \mu\left(Q_{i}\right)\right]\|g\|_{*} \leq C K_{Q_{i}, R} \mu\left(\rho Q_{i}\right)\|g\|_{*} .
\end{aligned}
$$

From (3.5), (3.6) and the definition of $b$, it follows that

$$
\left|\int_{R} b\left(g-g_{R}\right) d \mu\right| \leq \sum_{i}\left|\lambda_{i}\right|\left\|a_{i}\right\|_{L^{\infty}(\mu)} \int_{Q_{i}}\left|g-g_{R}\right| \leq C\|b\|_{h_{a^{a b b}}^{1, \infty}(\mu)}\|g\|_{*} .
$$

Now (3.4), (3.7) and (3.3) tell us (3.2). 
We now prove that the converse inequality in Lemma 3.2 is also true.

LEMMA 3.3. If $g \in \operatorname{rbmo}(\mu)$, then $\left\|L_{g}\right\|_{h_{a b b}^{1.0 .}(\mu)^{*}} \sim\|g\|_{*}$

PROOF. By Lemma 3.2, we only need to prove that $\left\|L_{g}\right\|_{h_{a b}^{1, \infty}(\mu)^{*}} \geq C^{-1}\|g\|_{*}$. We prove this by showing that there exists some function $f \in h_{\text {atb }}^{1, \infty}(\mu)$ such that

$$
\left|L_{g}(f)\right| \geq C^{-1}\|g\|_{0}\|f\|_{h_{a b b}^{1, \infty}(\mu)} .
$$

For simplicity we assume that $\rho=2$. Let $\epsilon>0$ be some small constant which will be fixed later. There are two possibilities:

(1) There exists some doubling cube $Q \subset \mathbb{R}^{d}$ with $l(Q) \leq 1$ such that

$$
\int_{Q}\left|g-\alpha_{Q}(g)\right| d \mu \geq \epsilon\|g\|_{\circ} \mu(Q),
$$

or there exists some doubling cube $Q \subset \mathbb{R}^{d}$ with $l(Q)>1$ such that

$$
\left|\alpha_{Q}(g)\right| \geq \epsilon\|g\|_{0} .
$$

(2) For any doubling cube $Q \subset \mathbb{R}^{d}$ with $l(Q) \leq 1$, (3.8) does not hold and for any doubling cube $Q \subset \mathbb{R}^{d}$ with $l(Q)>1$, (3.9) does not hold.

Let us first see case (1). If (3.8) holds, by an argument similar to that in [13, page 116], we can find an $f \in h_{a t b}^{1, \infty}(\mu)$ such that

$$
\left|L_{g}(f)\right|=\left|\int_{\mathbf{R}^{d}} g f d \mu\right| \geq C^{-1} \epsilon\|g\|_{0}\|f\|_{h_{a b b}^{1, \infty}(\mu)} .
$$

If (3.9) holds, we take $f=\operatorname{sign} g / \mu(Q)$. Then $f \in h_{a t b}^{1, \infty}(\mu)$ and $\|f\|_{h_{a t b}^{1, \infty}(\mu)} \leq C$. By (2.41) and (3.9), we then have

$$
\left|L_{g}(f)\right|=\int_{Q}|g| d \mu / \mu(Q) \geq C^{-1} \epsilon\|g\|_{0}\|f\|_{h_{a b b}^{1 . \infty}(\mu)} .
$$

Thus, in case (1), our claim holds.

Now, let us consider case (2). We have two subcases.

(a) For any two doubling cubes $Q \subset R$ with $l(Q) \leq 1$,

$$
\left|\alpha_{Q}(g)-\alpha_{R}(g)\right| \leq \frac{1}{2} K_{Q, R}\|g\|_{0} .
$$

(b) There are doubling cubes $Q \subset R$ with $l(Q) \leq 1$,

$$
\left|\alpha_{Q}(g)-\alpha_{R}(g)\right|>\frac{1}{2} K_{Q, R}\|g\|_{\circ} .
$$


Let us first consider case (a) under assumption (2). In this case, by the definition of $\|g\|_{0}$, there exists some cube $Q$ with $l(Q) \leq 1$ such that

$$
\int_{Q}\left|g-\alpha_{\widetilde{Q}}(g)\right| d \mu \geq \frac{1}{2}\|g\|_{\circ} \mu(2 Q) .
$$

If $l(\widetilde{Q}) \leq 1$, then by the argument in [13, page 117], we know that if we choose $\epsilon>0$ small enough, then our claim holds. If $l(\widetilde{Q})>1$, we then choose

$$
f=\chi_{\left\{x \in Q: g(x)>\alpha_{\tilde{Q}}(g)\right\}}-\chi_{\left\{x \in Q: g(x)<\alpha_{\tilde{Q}}(g)\right\}} .
$$

Then supp $f \subset Q \subset \tilde{Q}$, and $\|f\|_{h_{a t b}^{1 . \infty}(\mu)} \leq \mu(2 Q) K_{Q . \tilde{Q}} \leq C \mu(2 Q)$. Moreover, since (3.9) does not hold for $\widetilde{Q}$, by (3.10), we have

$$
\begin{aligned}
\left|L_{g}(f)\right|=\left|\int_{Q} g f d \mu\right| & =\left|\int_{Q}\left[g-\alpha_{Q}(g)\right] f d \mu\right|-\left|\alpha_{Q}(g)\right| \mu(Q) \\
& \geq \int_{Q}\left|g-\alpha_{Q}(g)\right| d \mu-\epsilon\|g\|_{0} \mu(Q) \\
& \geq \frac{1}{4}\|g\|_{0} \mu(2 Q) \geq C^{-1}\|g\|_{0}\|f\|_{h_{a t b}^{1 \infty}(\mu)},
\end{aligned}
$$

if we choose $\epsilon$ small enough. Thus, in this case, our claim also holds.

Let us consider case (b). If $l(R) \leq 1$, then the same argument as in [13, page 118] gives us the proof of our claim. If $l(R)>1$, we take $f=\chi_{Q}$. Then

$$
\|f\|_{h_{a b b}^{1, \infty}(\mu)} \leq \mu(2 Q) K_{Q, R} \leq C \mu(Q) K_{Q, R} .
$$

Since (3.8) does not hold for $Q$ and (3.9) does not hold for $R$, we then have

$$
\begin{aligned}
\left|L_{g}(f)\right| & =\left|\int_{Q} g f d \mu\right|=\left|\int_{Q}\left[g-\alpha_{Q}(g)\right] f d \mu+\alpha_{Q}(g) \mu(Q)\right| \\
& \geq\left|\alpha_{Q}(g)-\alpha_{R}(g)\right| \mu(Q)-\int_{Q}\left|g-\alpha_{Q}(g)\right| d \mu-\left|\alpha_{R}(g)\right| \mu(Q) \\
& \geq \frac{1}{2} K_{Q, R}\|g\|_{\circ} \mu(Q)-2 \epsilon\|g\|_{\circ} \mu(Q) \geq \frac{1}{4} K_{Q, R}\|g\|_{\circ} \mu(2 Q) \\
& \geq C^{-1}\|g\|_{\circ}\|f\|_{h_{a t b}^{1, \infty}(\mu)},
\end{aligned}
$$

if we choose $\epsilon$ small enough. Thus, in this case, our claim is still true.

To establish the duality between $h_{a t b}^{1, \infty}(\mu)$ and $\operatorname{rbmo}(\mu)$, we follow the same procedure as that for the duality between $H_{a t b}^{1, \infty}(\mu)$ and $R B M O(\mu)$ in [13]; see also [7]. To do so, we introduce the atom and block spaces $h_{a b b}^{1, p}(\mu)$ for $p \in(1, \infty)$ and we prove that they coincide with $h_{a t b}^{1, \infty}(\mu)$ and the dual of the space $h_{a t b}^{1, \infty}(\mu)$ is the space $r b m o(\mu)$ simultaneously.

In the following, for a fixed $\rho \in(1, \infty)$, a function $b \in L_{\text {loc }}^{1}(\mu)$ is called a $p$-atomic block if 
(i) there exists some cube $R$ such that $\operatorname{supp} b \subset R$,

(ii) $\int_{R} b d \mu=0$,

(iii) there are functions $a_{j}$ supported in cubes $Q_{j} \subset R$ and numbers $\lambda_{j} \in \mathbb{R}$ such that $b=\sum_{j=1}^{\infty} \lambda_{j} a_{j}$, and $\left\|a_{j}\right\|_{L^{p}(\mu)} \leq \mu\left(\rho Q_{j}\right)^{1 / p-1} K_{Q_{j}, R}^{-1}$.

A function $b \in L_{\text {loc }}^{1}(\mu)$ is called a $p$-block if it satisfies only (i) and (iii).

For a $p$-atomic block or a $p$-block, we define $|b|_{h_{a b}^{\prime . p}(\mu)}=\sum_{j}\left|\lambda_{j}\right|$. Then, we say that $f \in h_{a t b}^{1, p}(\mu)$ if there are $p$-atomic blocks or $p$-blocks $b_{i}$ such that

$$
f=\sum_{i} b_{i}
$$

with $\sum_{i}\left|b_{i}\right|_{h_{a b b}^{1, p}(\mu)}<\infty$, where $b_{i}$ when $\operatorname{supp} b_{i} \subset Q_{i}$ and $l\left(Q_{i}\right) \leq 1$ is a $p$-atomic block and $b_{i}$ when supp $b_{i} \subset Q_{i}$ and $l\left(Q_{i}\right)>1$ is a $p$-block. Moreover, we define the $h_{a t b}^{1, p}(\mu)$ norm of $f$ by $\|f\|_{h_{a t b}^{1, p}(\mu)}=\inf \sum_{i}\left|b_{i}\right|_{h_{a t b(}^{1, p}(\mu)}$, where the infimum is taken over all the possible decompositions of $f$ in $p$-atomic blocks and $p$-blocks as above.

If all the $b_{i}$ 's in (3.11) are $p$-atomic atoms, the all such $f$ 's form the space $H_{a t b}^{1, p}(\mu)$ which was first studied by Tolsa in [13]. Obviously, we have

$$
H_{a t b}^{1, p}(\mu) \subsetneq h_{a t b}^{1, p}(\mu) \subsetneq L^{1}(\mu) .
$$

The spaces $h_{a r b}^{1, p}(\mu)$ have similar properties to the spaces $h_{a t b}^{1, \infty}(\mu)$ (see Proposition 3.1). We omit the details of the proof (see also [13, Proposition 5.1]).

Proposition 3.4. Let $1<p<\infty$ and $1<\rho<\infty$.

(i) $h_{a t b}^{1, p}(\mu)$ is a Banach space.

(ii) $h_{a t b}^{1, p}(\mu) \subset L^{1}(\mu)$ with $\|f\|_{L^{1}(\mu)} \leq\|f\|_{h_{a t b}^{1, p}(\mu)}$.

(iii) For $1<p_{1} \leq p_{2} \leq \infty$, we have the continuous inclusion $h_{a b b}^{1, p_{2}}(\mu) \subset h_{a t b}^{1, p_{1}}(\mu)$.

(iv) The definition of $h_{\text {atb }}^{1, p}(\mu)$ does not dependent on the constant $\rho \in(1, \infty)$.

In the rest of this section, we assume the constant $\rho$ in the definition of the space $h_{a t b}^{1, p}(\mu)$ is 2 and the constants $\rho$ and $\eta$ in the definition of the space $r b m o(\mu)$ are also 2 . Moreover, for any $p \in(1, \infty)$, we define $p^{\prime}$ by $1 / p+1 / p^{\prime}=1$.

LEMMA 3.5. For any $p \in(1, \infty), \operatorname{rbmo}(\mu) \subset h_{a t b}^{1, p}(\mu)^{*}$. That is, for any $g \in$ $\operatorname{rbmo}(\mu)$, the linear functional $L_{g}(f)=\int_{\mathbf{R}^{d}} f g d \mu$ defined on bounded functions $f$ with compact support extends to a unique continuous linear functional $L_{g}$ on $h_{a t b}^{1, p}(\mu)$ with $\left\|L_{g}\right\|_{h_{\text {atb }}^{\prime \prime p}(\mu)^{*}} \leq C\|g\|_{*}$.

PROOF. Similar to the proof of Lemma 3.2, we only need to prove that if $b$ is a $p$-atomic block or $p$-block and $g \in \operatorname{rbmo}(\mu)$, then

$$
\left|\int_{\mathbb{R}^{d}} f g d \mu\right| \leq C|b|_{h_{a b b}^{1, p}(\mu)}\|g\|_{*} \text {. }
$$


In the following, we suppose $\operatorname{supp} b \subset R$ and $b=\sum_{j} \lambda_{j} a_{j}$. If $l(R) \leq 1$, then $b$ is a $p$-atomic block. Since $\int_{R} b d \mu=0$, then

$$
\begin{aligned}
\left|\int_{\mathbb{R}^{d}} b g d \mu\right| & =\left|\int_{R} b\left(g-m_{\tilde{R}}(g)\right) d \mu\right| \\
& \leq \sum_{i}\left|\lambda_{i}\right|\left\|a_{j}\right\|_{L^{p}(\mu)}\left\{\int_{Q_{j}}\left|g-m_{\tilde{R}}(g)\right|^{p^{\prime}} d \mu\right\}^{1 / p^{\prime}} .
\end{aligned}
$$

Since $g \in r b m o(\mu)$, by Corollary 2.9 , we have $g \in r b m o^{p^{\prime}}(\mu)$. From $l\left(Q_{i}\right) \leq l(R) \leq 1$, by (2.36) and (2.7), we deduce

$$
\begin{aligned}
\left\{\int_{Q_{j}}\left|g-m_{\tilde{R}}(g)\right|^{p^{\prime}} d \mu\right\}^{1 / p^{\prime}} \\
\quad \leq\left\{\int_{Q_{j}}\left|g-m_{\widetilde{Q}_{j}}(g)\right|^{p^{\prime}} d \mu\right\}^{1 / p^{\prime}}+\left|m_{\widetilde{Q_{j}}}(g)-m_{\tilde{R}}(g)\right| \mu\left(Q_{j}\right)^{1 / p^{\prime}} \\
\leq C\|g\|_{*} \mu\left(2 Q_{j}\right)^{1 / p^{\prime}}+C K_{Q_{j}, R}\|g\|_{*} \mu\left(Q_{j}\right)^{1 / p^{\prime}} \\
\quad \leq C K_{Q_{j}, R}\|g\|_{*} \mu\left(2 Q_{j}\right)^{1 / p^{\prime}} .
\end{aligned}
$$

From (3.13) and the definition of $p$-atomic blocks, we then deduce

$$
\left|\int_{\mathbb{R}^{d}} b g d \mu\right| \leq C \sum_{j}\left|\lambda_{j}\right|\|g\|_{*} \leq C|b|_{h_{a b b}^{1, p}(\mu)}\|g\|_{*} .
$$

If $l(R)>1$, then $b$ is a $p$-block. We then have

$$
\left|\int_{\mathbb{R}^{d}} b g d \mu\right| \leq\left|\int_{R} b\left[g-m_{\tilde{R}}(g)\right] d \mu\right|+\left|m_{\widetilde{R}}(g)\right| \int_{R}|b| d \mu .
$$

We first have

$$
\begin{aligned}
\left|m_{\tilde{R}}(g)\right| \int_{R}|b| d \mu & \leq C\|g\|_{*} \sum_{j}\left|\lambda_{j}\right| \int_{Q_{j}}\left|a_{j}\right| d \mu \\
& \leq C\|g\|_{*} \sum_{j}\left|\lambda_{j}\right|\left\|a_{j}\right\|_{L^{p}(\mu)} \mu\left(Q_{j}\right)^{1 / p^{\prime}} \\
& \leq C\|g\|_{*} \sum_{j}\left|\lambda_{j}\right| \leq C\|g\|_{*}\|b\|_{h_{a b}^{1, p}(\mu)} .
\end{aligned}
$$

If $l\left(Q_{j}\right) \leq 1$, by (2.36) and Remark 2.3, we easily see that (3.14) still holds. From this, it is easy to deduce

$$
\left|\int_{Q_{j}} a_{j}\left[g-m_{\widetilde{R}}(g)\right] d \mu\right| \leq\left\|a_{j}\right\|_{L^{p}(\mu)}\left\{\int_{Q_{j}}\left|g-m_{\widetilde{R}}(g)\right|^{p^{\prime}} d \mu\right\}^{1 / p^{\prime}} \leq C\|g\|_{*} .
$$


If $l\left(Q_{j}\right)>1$, by (2.37), we have

$$
\begin{aligned}
\left\{\int_{Q_{j}}\left|g-m_{\tilde{R}}(g)\right|^{p^{\prime}} d \mu\right\}^{1 / p^{\prime}} & \leq\left\{\int_{Q_{j}}|g|^{p^{\prime}} d \mu\right\}^{1 / p^{\prime}}+\left|m_{\widetilde{R}}(g)\right| \mu\left(Q_{j}\right)^{1 / p^{\prime}} \\
& \leq C\|g\|_{*} \mu\left(2 Q_{j}\right)^{1 / p^{\prime}}+C\|g\|_{*} \mu\left(Q_{j}\right)^{1 / p^{\prime}} \\
& \leq C\|g\|_{*} \mu\left(2 Q_{j}\right)^{1 / p^{\prime}},
\end{aligned}
$$

and therefore, $\left|\int_{Q_{j}} a_{j}\left[g-m_{\tilde{R}}(g)\right] d \mu\right| \leq C\|g\|_{*}$. By combining (3.16) and (3.17) with (3.15), we obtain our claim.

From the above, (3.12), and [13, Lemma 5.4], Lemma 3.4 follows; see also [7].

LEMMA 3.6. For $1<p<\infty$, we have $h_{a b b}^{1, p}(\mu)^{*} \subset L_{\mathrm{loc}}^{p^{\prime}}(\mu)$.

LEMMA 3.7. For $1<p<\infty$, we have $h_{a t b}^{1, p}(\mu)^{*} \subset \operatorname{rbmo}(\mu)$.

Proof. Let $g \in h_{\text {atb }}^{1, p}(\mu)^{*}$. By Lemma 3.7, we can suppose $g \in L_{\text {loc }}^{p^{\prime}}(\mu)$. We now verify that $g \in \operatorname{rbmo}(\mu)$ and $\|g\|_{0} \leq C\left\|L_{g}\right\|_{h_{a b b}^{1, p}(\mu)^{*}}$. We prove that for any cube $Q$ with $l(Q) \leq 1$,

$$
\begin{aligned}
\frac{1}{\mu(2 Q)} \int_{Q}\left|g-\alpha_{\tilde{Q}}(g)\right| d \mu & \leq C\left\|L_{g}\right\|_{h_{a b}^{1, p}(\mu)^{*}}, \\
\left|\alpha_{Q}(g)-\alpha_{R}(g)\right| & \leq C K_{Q, R}\left\|L_{g}\right\|_{h_{a b b}^{h, p}(\mu)^{*}}
\end{aligned}
$$

for any two doubling cubes $Q \subset R$ with $l(Q) \leq 1$, and for any doubling cube $Q$ with $l(Q)>1$,

$$
\left|\alpha_{Q}(g)\right| \leq C\left\|L_{g}\right\|_{h_{a t b}^{1, p}(\mu)^{*}} .
$$

We first prove (3.20). Let $Q$ be a doubling cube with $l(Q)>1$ and let $f=\chi_{Q} \operatorname{sign} g$. Then $f \in h_{a t b}^{1, p}(\mu)$ and $\|f\|_{h_{a b b}^{1, p}(\mu)} \leq C \mu(Q)$. By (2.41), we then obtain

$$
\begin{aligned}
\left|\alpha_{Q}(g)\right| & \leq \frac{2}{\mu(Q)} \int_{Q}|g| d \mu=\frac{2}{\mu(Q)}\left|\int_{Q} g f d \mu\right| \\
& \leq \frac{2}{\mu(Q)}\left\|L_{g}\right\|_{h_{a b b}^{1 . p}(\mu)^{*}}\|f\|_{h_{a b b}^{1, p}(\mu)} \leq C\left\|L_{g}\right\|_{h_{a b b}^{1, p}(\mu)^{\circ}} .
\end{aligned}
$$

Thus (3.20) holds.

We now verify (3.18). If $Q$ is a doubling cube, by the proof of [13, (5.2)] we know that (3.18) holds. Suppose now $Q$ is non doubling. If $l(\widetilde{Q}) \leq 1$, then (3.18) is also true by the proof of $[13,(5.2)]$. Now suppose that $l(\widetilde{Q})>1$. We take

$$
b=\frac{\left|g-\alpha_{\tilde{Q}}(g)\right|^{p^{\prime}}}{g-\alpha_{\tilde{Q}}(g)} \chi_{Q \cap\{g \neq \alpha \widetilde{\tilde{Q}}(g)\}} .
$$


Then supp $b \subset \widetilde{Q}$. By Lemma 1.3, we have $K_{Q, \tilde{Q}} \leq C$ and from this, it follows that

$$
\|b\|_{h_{a b b}^{1, p}(\mu)} \leq C\left\{\int_{Q}|g-\alpha \tilde{Q}(g)|^{p^{\prime}} d \mu\right\}^{1 / p} \mu(2 Q)^{1 / p^{\prime}} .
$$

Since (3.20) is true for $\widetilde{Q}$, by (3.21) and Proposition 3.4 (ii), we have

$$
\begin{aligned}
\int_{Q}\left|g-\alpha_{\tilde{Q}}(g)\right|^{p^{\prime}} d \mu & =\int_{Q}\left(g-\alpha_{\tilde{Q}}(g)\right) b d \mu \\
& \leq\left|\int_{Q} g b d \mu\right|+\left|\alpha_{\tilde{Q}}(g)\right| \int_{Q}|b| d \mu \\
& \leq C\left\|L_{g}\right\|_{h_{a b b}^{1, p}(\mu)^{*}}\|b\|_{h_{a b b}^{1, p}(\mu)} \\
& \leq C\left\|L_{g}\right\|_{h_{a b b}^{1, p}(\mu)^{*}}\left\{\int_{Q}\left|g-\alpha_{\tilde{Q}}(g)\right|^{p^{\prime}} d \mu\right\}^{1 / p^{\prime}} \mu(2 Q)^{1 / p^{\prime}} .
\end{aligned}
$$

That is,

$$
\left\{\frac{1}{\mu(2 Q)} \int_{Q}\left|g-\alpha_{\widetilde{Q}}(g)\right|^{p^{\prime}} d \mu\right\}^{1 / p^{\prime}} \leq C\left\|L_{g}\right\|_{h_{a t b}^{1, p}(\mu)} .
$$

which implies (3.18). Thus, in all the cases, we have (3.18) holds.

Finally, let us verify (3.19). If $l(R) \leq 1$, then by the proof of [13, (5.3)] we can easily obtain (3.19). Now suppose that $l(Q) \leq 1<l(R)$. We then take

$$
b=\frac{\left|g-\alpha_{R}(g)\right|^{p^{\prime}}}{g-\alpha_{R}(g)} \chi_{Q n\left(g \neq \alpha_{R}(g)\right)} .
$$

Then it is easy to check that

$$
\|b\|_{h_{a l b}^{\prime, p}(\mu)} \leq C K_{Q, R}\left\{\int_{Q}\left|g-\alpha_{R}(g)\right|^{p^{\prime}} d \mu\right\}^{1 / p} \mu(2 Q)^{1 / p^{\prime}}
$$

(see the proof of (3.21)). By (3.20) for $R$ and (3.23), as in (3.22), we then have

$$
\begin{aligned}
\int_{Q}\left|g-\alpha_{R}(g)\right|^{p^{\prime}} d \mu & \leq C\left\|L_{g}\right\|_{h_{a b}^{1, p}(\mu)} \cdot\|b\|_{h_{a b b}^{1, p}(\mu)} \\
& \leq C K_{Q, R}\left\|L_{g}\right\|_{h_{a b}^{1, p}(\mu)^{*}} \cdot\left\{\int_{Q}\left|g-\alpha_{R}(g)\right|^{p^{\prime}} d \mu\right\}^{1 / p} \mu(Q)^{1 / p^{\prime}} .
\end{aligned}
$$

Thus

$$
\left\{\frac{1}{\mu(Q)} \int_{Q}\left|g-\alpha_{R}(g)\right|^{p^{\prime}} d \mu\right\}^{1 / p^{\prime}} \leq C K_{Q, R}\left\|L_{g}\right\|_{h_{a b}^{1, p}(\mu)^{*}}
$$


Since $Q$ is doubling with $l(Q) \leq 1$, and, therefore, satisfies (3.18), by (3.24) we obtain

$$
\begin{aligned}
\left|\alpha_{Q}(g)-\alpha_{R}(g)\right| & =\frac{1}{\mu(Q)} \int_{Q}\left|\alpha_{Q}(g)-\alpha_{R}(g)\right| d \mu \\
& \leq \frac{1}{\mu(Q)} \int_{Q}\left|g-\alpha_{Q}(g)\right| d \mu+\frac{1}{\mu(Q)} \int_{Q}\left|\alpha_{R}(g)-g\right| d \mu \\
& \leq C K_{Q, R}\left\|L_{g}\right\|_{h_{a b b}^{1, p}(\mu)}{ }^{*} .
\end{aligned}
$$

That is, (3.19) also holds.

Now, if we replace [13, Lemmas 5.2, 5.3 and 5.4], respectively, by Lemmas 3.5, 3.7 and 3.6, then by the same argument as in [13, Theorem 5.5], we can prove the following theorem; see also [7]. We omit the details.

THEOREM 3.8. For $1<p<\infty, h_{a t b}^{1, p}(\mu)=h_{a t b}^{1, \infty}(\mu)$. Moreover, $h_{a t b}^{1, \infty}(\mu)^{*}=r b m o(\mu)$.

\section{An interpolation result}

In this section we choose $\eta=3 / 2$ and $\rho=2$ in the definition of the space $r b m o(\mu)$.

In [13] Tolsa has introduced the following sharp maximal operator related to the space $R B M O(\mu)$ :

$$
\begin{aligned}
M^{\sharp} f(x)= & \sup _{Q \ni x} \frac{1}{\mu\left(\frac{3}{2} Q\right)} \int_{Q}\left|f-m_{\tilde{Q}} f\right| d \mu \\
& +\sup _{Q \subset R: x \in Q, Q, R \text { doubling }} \frac{\left|m_{Q} f-m_{R} f\right|}{K_{Q, R}} .
\end{aligned}
$$

Then it is clear that $f \in R B M O(\mu)$ if and only if $M^{\sharp} f \in L^{\infty}(\mu)$. The sharp maximal operator related to our space $r b m o(\mu)$ is defined by

$$
\begin{aligned}
\tilde{M}^{\sharp} f(x)= & \sup _{Q \ni x, l(Q) \leq 1} \frac{1}{\mu\left(\frac{3}{2} Q\right)} \int_{Q}\left|f-m_{\tilde{Q}} f\right| d \mu \\
& +\sup _{Q \subset R: x \in Q, l(Q) \leq 1, Q, R \text { doubling }} \frac{\left|m_{Q} f-m_{R} f\right|}{K_{Q, R}} \\
& +\sup _{Q \ni x_{0} /(Q)>1} \frac{1}{\mu\left(\frac{3}{2} Q\right)} \int_{Q}|f| d \mu .
\end{aligned}
$$

Obviously, we have that $f \in \operatorname{rbmo}(\mu)$ if and only if $\tilde{M}^{\sharp} f \in L^{\infty}(\mu)$, and by (4.1)(4.2), we obtain

$$
M^{\sharp} f(x) \leq C \tilde{M}^{\sharp} f(x) .
$$


In [13], for $\rho \in(1, \infty)$, Tolsa also introduced the following maximal operators $N$ and $M_{(\rho)}$ :

$$
N f(x)=\sup _{Q \ni x, Q \text { doubling }} \frac{1}{\mu(Q)} \int_{Q}|f| d \mu \quad \text { and } \quad M_{(\rho)} f(x)=\sup _{Q \ni x} \frac{1}{\mu(Q)} \int_{Q}|f| d \mu .
$$

Tolsa proved that $N$ and $M_{(\rho)}$ are of weak type $(1,1)$ and bounded on $L^{p}(\mu), p \in$ $(1, \infty]$.

From (4.2) it is easy to see that $\tilde{M}^{\sharp} f(x) \leq C\left(M_{(3 / 2)} f(x)+N f(x)\right)$, which tells us that $\widetilde{M}^{\sharp}$ is of weak type $(1,1)$ and bounded on $L^{p}(\mu), p \in(1, \infty]$.

By (4.3) and [13, Theorem 6.2], we easily obtain the following lemma.

LEMMA 4.1. Let $f \in L_{\mathrm{loc}}^{1}(\mu)$ with $\int_{\mathbb{R}^{d}} f d \mu=0$ if $\|u\|<\infty$. For $1<p<\infty$, if $\inf (1, N f) \in L^{p}(\mu)$, then we have $\|N f\|_{L^{p}(\mu)} \leq C\left\|M^{\sharp} f\right\|_{L^{p}(\mu)} \leq C\left\|\tilde{M}^{\sharp} f\right\|_{L^{p}(\mu)}$.

Our main theorem in this section is the following interpolation result for operators.

THEOREM 4.2. Let T be a linear operator which is bounded from $h_{\text {atb }}^{1, \infty}(\mu)$ into $L^{1}(\mu)$ and from $L^{\infty}(\mu)$ into $r b m o(\mu)$. Then $T$ extends boundedly to $L^{p}(\mu), 1<p<\infty$.

The proof of Theorem 4.2 follows the scheme of the proof of [13, Theorem 7.2]; see also [7]. We need the following Calderón-Zygmund type decomposition, which is [13, Lemma 7.3].

LEMMA 4.3. For $1 \leq p<\infty$, consider $f \in L^{p}(\mu)$ with compact support. For any $\lambda>0$ (with $\lambda>\beta_{2}\|f\|_{L^{\prime}(\mu)} /\|\mu\|$ if $\left.\|\mu\|<\infty\right)$, we have

(a) There exists a finite of almost disjoint (namely, with a bounded overlap) cubes $\left\{Q_{i}\right\}_{i}$ such that

$$
\frac{1}{\mu\left(2 Q_{i}\right)} \int_{Q_{i}}|f|^{p} d \mu>\frac{\lambda^{p}}{\beta_{2}}, \quad \frac{1}{\mu\left(2 \eta Q_{i}\right)} \int_{\eta Q_{i}}|f|^{p} d \mu \leq \frac{\lambda^{p}}{\beta_{2}} \quad \text { for all } \eta>2,
$$

(b) For each $i$, let $w_{i}=\chi_{Q_{i}} /\left(\sum_{k} \chi_{Q_{k}}\right)$ and let $R_{i}$ be a $\left(6,6^{n+1}\right)$-doubling cube concentric with $Q_{i}$ and with $l\left(R_{i}\right)>4 l\left(Q_{i}\right)$. Then there exists a family of functions $\varphi_{i}$ with $\operatorname{supp} \varphi_{i} \subset R_{i}$ satisfying $\int_{R_{i}} \varphi_{i} d \mu=\int_{Q_{i}} f w_{i} d \mu$ and

$$
\sum_{i}\left|\varphi_{i}\right| \leq B \lambda
$$

(where $B$ is some constant), and if $1<p<\infty$,

$$
\left(\int_{R_{i}}\left|\varphi_{i}\right|^{p} d \mu\right)^{1 / p} \mu\left(R_{i}\right)^{1 / p^{\prime}} \leq \frac{C}{\lambda^{p-1}} \int_{Q_{i}}|f|^{p} d \mu .
$$


(c) For $1<p<\infty$, if $R_{i}$ is the smallest $\left(6,6^{n+1}\right)$-doubling cube of the family $\left\{6^{k} Q_{i}\right\}_{k \geq 1}$ and we set $b=\sum_{i}\left(f w_{i}-\varphi_{i}\right)$, then

$$
\|b\|_{H_{a b i}^{1, p}(\mu)} \leq \frac{C}{\lambda^{p-1}}\|f\|_{L^{p}(\mu)}^{p}
$$

PROOF OF THEOREM 4.2. For simplicity we assume $\|\mu\|=\infty$. Obviously, the functions $f \in L^{\infty}(\mu)$, having compact support, are dense in $L^{p}(\mu), 1<p<\infty$. For such functions we prove that

$$
\left\|\tilde{M}^{\sharp} T f\right\|_{L^{p}(\mu)} \leq C\|f\|_{L^{p}(\mu)}, \quad 1<p<\infty .
$$

By (4.7), Lemma 4.1 and the fact that $|f(x)| \leq N f(x)$ for $\mu$-a.e. $x \in \mathbb{R}^{d}$, we have $\|T f\|_{L^{p}(\mu)} \leq C\|f\|_{L^{p}(\mu)}$. Note that if $f \in L^{\infty}(\mu)$ has compact support, then $f \in$ $h_{a t b}^{1, \infty}(\mu)$ and $T f \in L^{1}(\mu)$. Thus $N(T f) \in L^{1, \infty}(\mu)$ and then inf $\{1, N(T f)\} \in L^{p}(\mu)$. This means the hypotheses of Lemma 4.1 are satisfied.

Given any function $f \in L^{p}(\mu), 1<p<\infty$, for $\lambda>0$, we take a family of almost disjoint cubes $\left\{Q_{i}\right\}_{i}$ as in Lemma 4.3 , and a collection of cubes $\left\{R_{i}\right\}_{i}$ as in (c) of Lemma 4.3. Then

$$
f=b+g=\sum_{i}\left(\frac{\chi_{Q_{i}}}{\sum_{k} \chi_{Q_{k}}} f-\varphi_{i}\right)+g .
$$

By (4.4)-(4.5), we have $\|g\|_{L^{\infty}(\mu)} \leq C \lambda$, and by (4.6) and (3.12),

$$
\|b\|_{h_{a b b}^{1, p}(\mu)} \leq\|b\|_{H_{a i b}^{1, p}(\mu)} \leq \frac{C}{\lambda^{p-1}}\|f\|_{L^{p}(\mu)}^{p} .
$$

Since $T$ is bounded from $L^{\infty}(\mu)$ into $r b m o(\mu)$, we have $\left\|\tilde{M}^{\sharp} T f\right\|_{L^{\infty}(\mu)} \leq C_{10} \lambda$. Thus

$$
\left\{x \in \mathbb{R}^{d}: \tilde{M}^{\sharp} T f(x)>\left(C_{10}+1\right) \lambda\right\} \subset\left\{x \in \mathbb{R}^{d}: \tilde{M}^{\sharp} T b(x)>\lambda\right\} .
$$

Since $\widetilde{M}^{\sharp}$ is of weak type $(1,1)$, we have

$$
\mu\left\{x \in \mathbb{R}^{d}: \tilde{M}^{\sharp} T b(x)>\lambda\right\} \leq C \frac{\|T b\|_{L^{\prime}(\mu)}}{\lambda} .
$$

On the other hand, as $T$ is bounded from $h_{\text {atb }}^{1, \infty}(\mu)$ into $L^{1}(\mu)$,

Thus

$$
\|T b\|_{L^{\prime}(\mu)} \leq C\|b\|_{h_{a i b}^{1 \infty}(\mu)} \leq \frac{C}{\lambda^{p-1}}\|f\|_{L^{p}(\mu)}^{p} .
$$

$$
\mu\left\{x \in \mathbb{R}^{d}: \tilde{M}^{\sharp} T f(x)>\lambda\right\} \leq \frac{C}{\lambda^{p}}\|f\|_{L^{p}(\mu)}^{p} .
$$

This means that the sublinear operator $\tilde{M}^{\sharp} T$ is of weak type $(p, p)$ for all $p \in(1, \infty)$. By the Marcinkiewicz interpolation theorem we obtain that $\widetilde{M}^{\sharp} T$ is bounded on $L^{p}(\mu)$ for all $p \in(1, \infty)$. In particular, (4.7) holds for a bounded function $f$ with compact support. Thus our claim holds. 


\section{Relation between $h_{a t b}^{1, \infty}(\mu)$ and $H_{a t b}^{1, \infty}(\mu)$}

In this section, we establish some relation between the space $h_{a t b}^{1, \infty}(\mu)$ and the space $H_{a b b}^{1, \infty}(\mu)$ studied in [13]. Let us first recall the characterization of the Hardy space $H_{a b b}^{1, \infty}(\mu)$ in terms of a maximal operator $M_{\Phi}$ which was established in [15] and will be used in the following.

DEFINITION 5.1. Given $f \in L_{\mathrm{loc}}^{1}(\mu)$, we set

$$
M_{\Phi} f(x)=\sup _{\varphi \sim x}\left|\int_{\mathbb{R}^{d}} f \varphi d \mu\right| .
$$

where the notation $\varphi \sim x$ means that $\varphi \in L^{1}(\mu) \cap C^{1}\left(\mathbb{R}^{d}\right)$ and satisfies

(i) $\|\varphi\|_{L^{1}(\mu)} \leq 1$,

(ii) $0 \leq \varphi(y) \leq 1 /|y-x|^{n}$ for all $y \in \mathbb{R}^{d}$, and

(iii) $|\nabla \varphi(y) \leq 1 /| y-\left.x\right|^{n+1}$ for all $y \in \mathbb{R}^{d}$.

LEMMA 5.2. A function $f$ belongs to the space $H_{a t b}^{1, \infty}(\mu)$ if and only if $f \in L^{1}(\mu)$, $\int_{\mathbb{R}^{d}} f d \mu=0$ and $M_{\Phi} f \in L^{1}(\mu)$. Moreover, in this case,

$$
\|f\|_{H_{a b h}^{1 ; \infty}(\mu)} \sim\|f\|_{L^{1}(\mu)}+\left\|M_{\Phi} f\right\|_{L^{\prime}(\mu)} .
$$

To establish the relation between the space $h_{a t b}^{1, \infty}(\mu)$ and the space $H_{a t b}^{1, \infty}(\mu)$ we need a special function. Let $\phi$ be a function on $\mathbb{R}^{d} \times \mathbb{R}^{d}$ satisfying

$$
\begin{aligned}
& \phi(x, y)=0 \text { if }|x-y| \geq C_{\phi}, \text { where } C_{\phi}>0 \text { is a constant; } \\
& |\phi(x, y)| \leq C_{\phi} ; \\
& \text { for } \mu \text {-a.e. } y \in \operatorname{supp} \mu, \quad \int_{\mathbb{R}^{d}} \phi(x, y) d \mu(x)=1 .
\end{aligned}
$$

Obviously, if supp $\mu$ is bounded, we can just take

$$
\phi(x, y)=\frac{1}{\mu(\operatorname{supp} \mu)} \chi_{\text {supp } \mu}(x) \chi_{\operatorname{supp} \mu}(y) .
$$

Then this function $\phi$ satisfies (5.1)-(5.3). Moreover, if $\phi$ satisfies (5.1)-(5.3), then $\phi$ also has the following properties:

(5.4) $|\phi(x, y)| \leq \frac{C_{\phi}}{|x-y|^{n}} \quad$ for $x \neq y$;

(5.5) for $\mu$-a.e. $y \in \operatorname{supp} \mu, \quad \int_{\mathbb{R}^{d}}|\phi(x, y)| d \mu(x)+\int_{\mathbb{R}^{d}}|\phi(y, x)| d \mu(x) \leq C_{\phi}$. 
In the following, for a function $\phi$ on $\mathbb{R}^{d} \times \mathbb{R}^{d}$ and suitable functions $f$, we define

$$
T_{\phi} f(x)=\int_{\mathbb{R}^{d}} \phi(x, y) f(y) d \mu(y) .
$$

THEOREM 5.3. Let $\phi$ be a function on $\mathbb{R}^{d} \times \mathbb{R}^{d}$ satisfying (5.1)-(5.3). If $\in h_{\text {atb }}^{1, \infty}(\mu)$, then $f-T_{\phi} f \in H_{a t b}^{1, \infty}(\mu)$ and $\left\|f-T_{\phi} f\right\|_{H_{a b b}^{1, \infty}(\mu)} \leq C\|f\|_{h_{a b b}^{1, \infty}(\mu)}$, where $C$ is independent of $f$.

PROOF. By the definition of the space $h_{a t b}^{1, \infty}(\mu)$ and Lemma 5.2, to prove Theorem 5.3, we only need to verify that if $b$ is a block or an atomic block, say, $b=\sum \lambda_{j} a_{j}$, then $\int_{\mathbb{R}^{d}} M_{\Phi}\left(b-T_{\phi} b\right)(x) d \mu(x) \leq C \sum_{j}\left|\lambda_{j}\right|$. Let $\operatorname{supp} b \subset R$, where $R$ is a cube with the center $z_{R}$ and the side length $l(R)$.

We first suppose $b$ is a block. Then $l(R)>1$. In this case, it is easy to see that supp $T_{\phi} b \subset C_{11} R$, where $C_{11} \geq 0$ depends only on $C_{\phi}$. We then write

$$
\int_{\mathbb{R}^{d}} M_{\Phi}\left(b-T_{\phi} b\right)(x) d \mu(x)=\int_{\mathbb{R}^{d} \backslash 2 C_{11} R} M_{\Phi}\left(b-T_{\phi} b\right)(x) d \mu(x)+\int_{2 C_{11} R} \cdots=I_{1}+I_{2} .
$$

For $I_{1}$, by (5.5) and $\int_{R^{d}}\left[b(y)-T_{\phi} b(y)\right] d \mu(y)=0$, we obtain that, when $x \in$ $\mathbb{R}^{d} \backslash 2 C_{11} R$, for $\varphi \sim x$,

$$
\begin{aligned}
\mid \int_{\mathbb{R}^{d}}[ & \left.b(y)-T_{\phi} b(y)\right] \varphi(y) d \mu(y) \mid \\
& =\left|\int_{\mathbb{R}^{d}}\left[b(y)-T_{\phi} b(y)\right]\left[\varphi(y)-\varphi\left(z_{R}\right)\right] d \mu(y)\right| \\
& \leq \frac{C l(R)}{\left|x-z_{R}\right|^{n+1}}\left[\int_{\mathbb{R}^{d}}|b(y)| d \mu(y)+\int_{\mathbb{R}^{d}} \int_{\mathbb{R}^{d}}|\phi(y, z)||b(z)| d \mu(z) d \mu(y)\right] \\
& \leq \frac{C l(R)}{\left|x-z_{R}\right|^{n+1}} \int_{\mathbb{R}^{d}}|b(y)| d \mu(y) \\
& \leq \frac{C l(R)}{\left|x-z_{R}\right|^{n+1}} \sum_{j}\left|\lambda_{j}\right| .
\end{aligned}
$$

From this, it follows that

$$
I_{1} \leq C \sum_{j}\left|\lambda_{j}\right| \int_{\mathbb{R}^{d} \backslash 2 C_{11} R} \frac{C l(R)}{\left|x-z_{R}\right|^{n+1}} d \mu(x) \leq C \sum_{j}\left|\lambda_{j}\right| .
$$

Now let us estimate $I_{2}$. We first have

$$
\begin{aligned}
I_{2} & \leq \int_{2 C_{11} R}\left[M_{\Phi} b(x)+M_{\Phi}\left(T_{\phi} b\right)(x)\right] d \mu(x) \\
& \leq \sum_{j}\left|\lambda_{j}\right|\left[\int_{2 C_{11} R} M_{\Phi} a_{j}(x) d \mu(x)+\int_{2 C_{11} R} M_{\Phi}\left(T_{\phi} a_{j}\right)(x) d \mu(x)\right] .
\end{aligned}
$$


To obtain a desired estimate for $I_{2}$, we need to prove

$$
\int_{2 C_{11} R} M_{\Phi} a_{j}(x) d \mu(x)+\int_{2 C_{11} R} M_{\Phi}\left(T_{\Phi} a_{j}\right)(x) d \mu(x) \leq C .
$$

Let $Q_{j}$ be the support of $a_{j}$ and $z_{Q_{j}}$ be its center. We further write

$$
\int_{2 C_{11} R} M_{\Phi} a_{j}(x) d \mu(x)=\int_{2 Q_{j}} M_{\Phi} a_{j}(x) d \mu(x)+\int_{2 C_{11} R \backslash 2 Q_{j}} \cdots .
$$

When $x \in 2 Q_{j}$ and $\varphi \sim x$, we have

$$
\left|\int_{\mathbb{R}^{d}} \varphi(y) a_{j}(y) d \mu(y)\right| \leq\left\|a_{j}\right\|_{L^{\infty}(\mu)}\|\varphi\|_{L^{1}(\mu)} \leq\left\|a_{j}\right\|_{L^{\infty}(\mu)} .
$$

From this and the definition of a block, it follows that

$$
\int_{2 Q_{j}} M_{\Phi} a_{j}(x) d \mu(x) \leq\left\|a_{j}\right\|_{L^{\infty}(\mu)} \mu\left(2 Q_{j}\right) \leq C .
$$

When $x \in 2 C_{11} R \backslash 2 Q_{j}$, by the definition of $\varphi \sim x$, we have

$$
\left|\int_{\mathbb{R}^{d}} \varphi(y) a_{j}(y) d \mu(y)\right| \leq \frac{1}{\left|x-z_{Q_{j}}\right|^{n}}\left\|a_{j}\right\|_{L^{L^{\prime}}(\mu)} .
$$

From this, Lemma 1.3 and the definition of a block, we deduce

$$
\begin{aligned}
\int_{2 C_{11} R \backslash 2 Q_{j}} M_{\Phi} a_{j}(x) d \mu(x) & \leq C\left\|a_{j}\right\|_{L^{1}(\mu)} \delta\left(2 Q_{j}, 2 C_{11} R\right) \\
& \leq C\left\|a_{j}\right\|_{L^{\infty}(\mu)} \mu\left(Q_{j}\right) K\left(Q_{j}, R\right) \leq C .
\end{aligned}
$$

The estimate for the second term in the left-hand side of (5.6) is similar. We also write

$$
\int_{2 C_{11} R} M_{\Phi}\left(T_{\phi} a_{j}\right)(x) d \mu(x)=\int_{2 Q_{j}} M_{\Phi}\left(T_{\phi} a_{j}\right)(x) d \mu(x)+\int_{2 C_{11} R \backslash 2 Q_{j}} \cdots
$$

For the first term, when $x \in 2 Q_{j}$, by (5.5) and the definition of $\varphi \sim x$, we have

$$
\left|\int_{\mathbb{R}^{d}} \varphi(y) T_{\phi} a_{j}(y) d \mu(y)\right| \leq \int_{\mathbb{R}^{d}} \int_{\mathbb{R}^{d}}\left|\varphi(y) \phi(y, z) a_{j}(z)\right| d \mu(z) d \mu(y) \leq C\left\|a_{j}\right\|_{L^{\infty}(\mu)} .
$$

From this, it follows that

$$
\int_{2 Q_{j}} M_{\Phi}\left(T_{\phi} a_{j}\right)(x) d \mu(x) \leq C\left\|a_{j}\right\|_{L^{\infty}(\mu)} \mu\left(2 Q_{j}\right) \leq C
$$


which is a desired estimate. When $x \in 2 C_{11} R \backslash 2 Q_{j}$ and $\varphi \sim x$, by (5.4)-(5.5), we have

$$
\begin{aligned}
& \left|\int_{\mathbb{R}^{d}} \varphi(y)\left(T_{\phi} a_{j}\right)(y) d \mu(y)\right| \\
& \quad \leq \int_{\mathbb{R}^{d}}\left|a_{j}(z)\right|\left[\int_{\mathbb{R}^{d}}|\varphi(y) \phi(y, z)| d \mu(y)\right] d \mu(z) \\
& \quad \leq \int_{\mathbb{R}^{d}}\left|a_{j}(z)\right|\left[\int_{|y-z| \geq \frac{1}{2}|x-z|}|\varphi(y) \phi(y, z)| d \mu(y)+\int_{|y-z|<\frac{1}{2}|x-z|} \cdots\right] d \mu(z) \\
& \quad \leq \frac{C}{\left|x-z_{Q_{j}}\right|^{n}}\left\|a_{j}\right\|_{L^{\prime}(\mu)} .
\end{aligned}
$$

Then similar to (5.7), we can prove $\int_{2 C_{11} R \backslash 2 Q_{j}} M_{\Phi}\left(T_{\phi} a_{j}\right)(x) d \mu(x) \leq C$ which is a desired estimate. Thus (5.6) is true. We have proved our claim when $b$ is a block.

Now let us prove our claim when $b$ is an atomic block. Suppose supp $b \subset R$ with the center $z_{R}$ and the side length $l(R)<1$. Let $R_{1}$ be the cube with the center $z_{R}$ and the side length $C_{\phi}+l(R)$. Then supp $T_{\phi} b \subset R_{1}$. We then write

$$
\begin{aligned}
\int_{\mathbb{R}^{d}} M_{\Phi}\left(b-T_{\phi} b\right)(x) d \mu(x) & =\int_{\mathbb{R}^{d} \backslash 2 R_{1}} M_{\Phi}\left(b-T_{\phi} b\right)(x) d \mu(x)+\int_{2 R_{1} \backslash 2 R} \cdots+\int_{2 R} \cdots \\
& =I_{3}+I_{4}+I_{5} .
\end{aligned}
$$

The estimate for $I_{3}$ is similar to $I_{1}$ by replacing $2 C_{11} R$ there by $R_{1}$ here. We omit the details. To estimate $I_{4}$, we further write it into

$$
I_{4} \leq \int_{2 R_{1} \backslash 2 R} M_{\Phi} b(x) d \mu(x)+\int_{2 R_{1} \backslash 2 R} M_{\Phi}\left(T_{\phi} b\right)(x) d \mu(x) .
$$

On the first term, by the definition of $\varphi \sim x$ and $\int_{\mathbb{R}^{d}} b(y) d \mu(y)=0$, we have that when $x \in 2 R_{1} \backslash 2 R$,

$$
\begin{aligned}
\left|\int_{\mathbb{R}^{d}} \varphi(y) b(y) d \mu(y)\right| & =\left|\int_{\mathbb{R}^{d}}\left[\varphi(y)-\varphi\left(z_{R}\right)\right] b(y) d \mu(y)\right| \\
& \leq \frac{C l(R)}{\left|x-z_{R}\right|^{n+1}}\|b\|_{L^{1}(\mu)} \leq \frac{C l(R)}{\left|x-z_{R}\right|^{n+1}} \sum_{j}\left|\lambda_{j}\right| .
\end{aligned}
$$

From this it follows that

$$
\int_{2 R_{1} \backslash 2 R} M_{\Phi} b(x) d \mu(x) \leq C l(R) \sum_{j}\left|\lambda_{j}\right| \int_{2 R_{1} \backslash 2 R} \frac{1}{\left|x-z_{R}\right|^{n+1}} d \mu(x) \leq C \sum_{j}\left|\lambda_{j}\right|,
$$


which is a desired estimate. For the second term in (5.8), by the definition of $\varphi \sim x$ and (5.2), we then have

$$
\left|\int_{\mathbb{R}^{d}} \varphi(y) T_{\phi} b(y) d \mu(y)\right| \leq C\|\varphi\|_{L^{1}(\mu)}\|b\|_{L^{1}(\mu)} \leq C \sum_{j}\left|\lambda_{j}\right| .
$$

We then deduce

$$
\int_{2 R_{1} \backslash 2 R} M_{\Phi}\left(T_{\phi} b\right)(x) d \mu(x) \leq C \sum_{j}\left|\lambda_{j}\right| \mu\left(2 R_{1}\right) \leq C \sum_{j}\left|\lambda_{j}\right|
$$

which is also a desired estimate. The estimate for $I_{5}$ can be obtained by a similar way to that for $I_{2}$. We only need to replace $2 C_{11} R$ there by $2 R$ here. We omit the details.

Let $\phi$ be a function satisfying (5.1)-(5.3). We then define

$$
T_{\phi}^{*} f(x)=\int_{\mathbb{R}^{d}} \phi(y, x) f(y) d \mu(y)
$$

for any suitable function $f$. Then, by a proof similar to that of $[4$, Corollary 1$]$, we can easily deduce the following corollary from Theorem 3.8 and Theorem 5.3. We omit the details.

COROLlaRY 5.4. Let $\phi$ be the same function as in Theorem 5.3. Then

$$
\operatorname{rbmo}(\mu)=\left\{b \in R B M O(\mu): T_{\phi}^{*} b \in L^{\infty}(\mu)\right\} .
$$

\section{Some remarks}

In this section, we further suppose the measure $\mu$ satisfying the following condition $\mu(B(x, r)) \sim r^{n}$ for all $x \in \operatorname{supp} \mu$ and all $0<r \leq \operatorname{diam}(\operatorname{supp} \mu)$, where, for a set $E$, $\operatorname{diam} E=\sup \{|x-y|: x, y \in E\}$. Then, $\operatorname{supp} \mu$ is a space of homogeneous type in the sense of Coifman and Weiss in [1].

Let $h^{1}(\mu)=F_{1}^{0,2}(\operatorname{supp} \mu)$ which is introduced in [6]. Then it is easy to see that in this case, we have $h^{1}(\mu)=h_{a t b}^{1, \infty}(\mu)$. In fact, by the atom and block decomposition of $F_{1}^{0,2}(\operatorname{supp} \mu)$ in [6], we can easily deduce

$$
F_{1}^{0,2}(\operatorname{supp} \mu) \subset h_{a t b}^{1,2}(\mu)=h_{a t b}^{1, \infty}(\mu)
$$

from Theorem 3.8. Conversely, from [13, (2.24) and (2.25)] it is also easy to see that each 2-atomic block or 2-block $b$ in Section 3 is just a $(1,2,0)$ atom or $(1,2,0)$ block $b_{1}$ for $F_{1}^{0,2}(\operatorname{supp} \mu)$ times a coefficients $C|b|_{h_{a i b}^{1,2}(\mu)}$. Thus

$$
h_{a t b}^{1, \infty}(\mu)=h_{a t b}^{1,2}(\mu) \subset F_{1}^{0,2}(\operatorname{supp} \mu) .
$$


That is, our claim holds. Moreover, by [13, Example 5.6] and [5, Theorem C], we know that $H_{a i b}^{1, \infty}(\mu)=\dot{F}_{1}^{0,2}(\operatorname{supp} \mu)$ which was introduced by Han in [5]. We remark that our Theorem 5.3 and Corollary 5.4 are also true for this special case. Moreover, the function $\phi$ satisfying (5.1)-(5.3) exists in this special case. In fact, we can just take $\phi=S_{k}$ for some fixed $k \in \mathbb{Z}$, where $\left\{S_{k}\right\}_{k \in \mathbb{Z}}$ is an approximation to the identity constructed by Coifman's method; see $[2,5,6]$.

\section{References}

[1] R. R. Coifman and G. Weiss, Analyse harmonique non-commutative sur certains espaces homogènes, Lecture Notes in Math. 242 (Springer, Berlin, 1971).

[2] G. David, J.-L. Journé and S. Semmes, 'Opérateurs de Calderón-Zygmund, fonctions para-accrétive et interpolation', Rev. Mat. Iberoamericana 1 (1985), 1-56.

[3] J. García-Cuerva and J. L. Rubio de Francia, Weighted norm inequalities and related topics, North-Holland Math. Studies 116 (North-Holland, Amsterdam, 1985).

[4] D. Goldberg, 'A local version of real Hardy spaces', Duke Math. 46 (1979), $27-42$.

[5] Y. Han, 'Triebel-Lizorkin spaces on spaces of homogeneous type', Studia Math. 108 (1994), 247-273.

[6] Y. Han, S. Lu and D. Yang, 'Inhomogeneous Triebel-Lizorkin spaces on spaces of homogeneous type', Math. Sci. Res. Hot-Line 3 (1999), 1-29.

[7] J.-L. Journé, Calderón-Zygmund operators, pseudo-differential operators and the Cauchy integral of Calderón, Lecture Notes in Math. 994 (Springer, Berlin, 1983).

[8] J. Mateu, P. Mattila, A. Nicolau and J. Orobitg, 'BMO for nondoubling measures', Duke Math. J. $102(2000), 533-565$.

[9] F. Nazarov, S.'Treil and A. Volberg, "Cauchy integral and Calderón-Zygmund operators on nonhomogeneous spaces', Internat. Math. Res. Notices 15 (1997), 703-726.

[10] _ - 'Weak type estimates and Cotlar inequalities for Calderón-Zygmund operators on nonhomogeneous spaces', Internat. Math. Res. Notices 9 (1998), 463-487.

[11] __ 'The Tb-theorem on non-homogeneous spaces', Acta Math. 190 (2003), 151-239.

[12] X. Tolsa, ' $L$ ' -boundedness of the Cauchy integral operator for continuous measures', Duke Math. J. 98 (1999), 269-304.

[13], ' $B M O, H^{1}$, and Calderón-Zygmund operators for non doubling measures', Math. Ann. $319(2001), 89-149$.

[14] - 'Littlewood-Paley theory and the $T(1)$ theorem with non doubling measures', Adv. Math. $164(2001), 57-116$.

[15] _ 'The space $H^{1}$ for non doubling measures in terms of a grand maximal operator', Trans. Amer. Math. Soc. 355 (2003), 315-348.

[16] J. Verdera, 'On the $T(1)$ theorem for the Cauchy integral', Ark. Mat. 38 (2000), 183-199.

\section{School of Mathematical Sciences}

Beijing Normal University

Beijing 100875

People's Republic of China

e-mail: dcyang@bnu.edu.cn 